\title{
THE CONSTRUCTION OF ANALYTIC DIFFEOMORPHISMS FOR EXACT ROBOT NAVIGATION ON STAR WORLDS
}

\author{
ELON RIMON AND DANIEL E. KODITSCHEK
}

\begin{abstract}
A Euclidean Sphere World is a compact connected submanifold of Euclidean $n$-space whose boundary is the disjoint union of a finite number of $(n-1)$ dimensional Euclidean spheres. A Star World is a homeomorph of a Euclidean Sphere World, each of whose boundary components forms the boundary of a star shaped set. We construct a family of analytic diffeomorphisms from any analytic Star World to an appropriate Euclidean Sphere World "model." Since our construction is expressed in closed form using elementary algebraic operations, the family is effectively computable. The need for such a family of diffeomorphisms arises in the setting of robot navigation and control. We conclude by mentioning a topological classification problem whose resolution is critical to the eventual practicability of these results.
\end{abstract}

\section{INTRODUCTION}

Consider the following problem in robotics. A kinematic chain-a sequence of mutually constrained actuated rigid bodies-is allowed to move in a cluttered workplace. Contained within the joint space - an analytic manifold which forms the configuration space of the kinematic chain-is the free space, $\mathscr{F}$-the set of all configurations which do not involve intersection with any of the "obstacles" cluttering the workspace. Given a "destination point" in the interior of $\mathscr{F}$ to which it is desired to move the robot, and an arbitrary initial point, find a curve in $\mathscr{F}$ joining the initial point with the desired destination.

Supposing that $\partial \mathscr{F}$ is a $C^{(1)}$ manifold, the negative gradient vector field of a scalar valued function which is transverse (exterior directed) on the boundary of the free space, and which has a single minimum at the destination point gives rise to a flow which moves almost all initial conditions toward that desired point. Thus, a suitably chosen scalar valued "cost" function solves the geometric problem of finding paths to the destination in free space. Moreover, interpreting the cost function as an artificial potential energy, it can be shown that a gradient vector field on $\mathscr{F}$ "lifts naturally" to a Lagrangian vector field

Received by the editors December 27, 1988.

1980 Mathematics Subject Classification (1985 Revision). Primary 70B15, 93A99; Secondary 93C10, 93015, 52A30.

This work was supported in part by the National Science Foundation under grant DMC-8505160, and, in part by a Presidential Young Investigator Award held by the second author. 
on the phase space of $\mathscr{F}, T \mathscr{F}$, describing the robot's Newtonian dynamics when subjected to a suitable feedback compensating control law [10]. Under certain additional regularity conditions, the Lagrangian system "inherits" the limit properties of the gradient system, and a portion of $T \mathscr{F}$, including the zero section, is positive invariant with respect to the lifted flow [9]. Thus, a further constrained cost function solves the robot navigation problem and the attendant control problems simultaneously.

The purely geometric problem of constructing a curve between two points in a space obstructed by sets with arbitrary polynomial boundary (given perfect information) has already been completely solved by Schwartz and Sharir [20]. Canny [3] has recently offered a much more efficient algorithm for this class of problems as well. The motivation for the present direction of inquiry (beyond its apparent academic interest) is the desire to incorporate explicitly aspects of the control problem - the construction of feedback compensators for a well characterized class of dynamical systems in the presence of well characterized constraints -in the planning phase of robot navigation problems. That is, the geometrical "find path" problem is generalized to the search for a family of paths in $\mathscr{F}$ (the one-parameter group of the gradient flow), which provides a feedback control law for the physical robot as well. The idea of using scalar valued functions for the specification of robot tasks with a view of the control problems in mind was pioneered by Khatib [8] in the context of obstacle avoidance. Fundamental work of Hogan [5] in the context of force control further advanced the interest in this approach. The methodology has been developed independently by Arimoto in Japan [1], and by Soviet investigators as well [18]. To the best of our knowledge, no attempt at exact global navigation using scalar valued functions has been offered heretofore.

Can it be guaranteed that such "navigation functions" exist? If they exist, can one find a computationally effective procedure for constructing them? The answer to the first question is an unequivocal "yes." In a recent paper [12], we have proposed a formal definition of a navigation function, and, using Smale's "nice" functions [22], we have shown that smooth navigation functions exist on any smooth manifold with boundary. Moreover, we have noted that the navigation properties are invariant under $C^{(q)}$ diffeomorphisms for $q \geq 1$ : that is to say, a navigation function on a given space induces a navigation function on every other space in its diffeomorphism equivalence class via composition (pull back). Finally, we have constructed a family of navigation functions on Euclidean sphere worlds: these are compact connected submanifolds of Euclidean $n$-sapce whose boundary is formed by the disjoint union of a finite number of Euclidean $(n-1)$-spheres.

The answer to the second question obviously depends upon the nature of freespace, $\mathscr{F}$, and the desired model of computation. In their pioneering solution to the geometric navigation problem, Schwartz and Sharir [20] have argued persuasively that the class of real algebraic functions on real semialgebraic vari- 
eties adequately models freespace and provides a practicable notion of "effective computation." We will adopt this point of view and insist that our constructions remain within the class of real algebraic functions: that is to say, the level set of a navigation function must be real semialgebraic. But since our applicationthe specification of a control law for a mechanical system-depends upon the gradient, we will further demand that navigation functions be smooth as well. Now the standard appeal to functions "patched" together through a smooth partition of unity will not avail: for this would involve transcendental expressions that depart form the desired model of computation via a finite number of elementary operations. We are confronted instead with the harder task of building analytic algebraic functions.

This paper presents a particular mathematical construction that we feel offers strong hope for the success of the general program. We consider the navigation problem on a specific subclass of analytic sphere worlds-the star worlds-each of whose members is a compact connected subset of $E^{n}$ obtained by removing from a compact star shaped set with analytic boundary a finite number of smaller disjoint open star shaped sets with analytic boundaries. Our previous Euclidean sphere world construction [12] yields an analytic algebraic navigation function-a scalar valued map formed by quotients of polynomials and a $k$ th root-whose complexity grows with the square of both the dimension of the embedding space, $n$, and the number of "obstacles," $M$. According to the results reported in that paper, the Star World navigation problem is immediately solved by the construction of an effectively computable diffeomorphism to a suitable Euclidean sphere world "model." Here, we present such a class of diffeomorphisms formed by the composition of a one-parameter family of analytic algebraic functions-rational functions and a square root-with "user supplied" boundary functions whose zero level sets describe the boundary components. The parameter is explicitly computed in closed form from geometric information about the relative locations of the star shaped sets. Thus, the complete construction is analytic and algebraic as long as the boundary functions are, themselves, analytic and algebraic.

This advances our program of research toward the goal of developing "geometric expressiveness" rich enough for navigation amidst real world obstacles. It is manifestly clear, however, that arbitrarily shaped and jointed robots operating amidst real world clutter will give rise to free spaces which are neither smooth nor deformed sphere worlds. A paper presently in preparation concerns the extension of these results to spaces in the topological equivalence class of the sphere worlds whose obstacles are comprised of finite unions of star shaped sets. We suspect that this class forms a dense subset of the topologically deformed sphere worlds. The more challenging question, by far, to the eventual practicability of our approach, is the general determination of a topological model for free space given a topological classification of the cluttered environment and a complete characterization of the robot. 
The paper is organized as follows. This introductory section continues with a formal statement of the problem at hand, and a specification of the assumptions concerning the available information. In the next section, we define the class of star worlds, and present an explicit one-parameter family of analytic functions defined on an arbitrary star world. In $\S 3$ we prove Theorem 1: for any star world, a lower bound on the parameter is specified in terms of the boundary locations, guaranteeing that each member of this family, whose parameter value complies with this bound, is an analytic diffeomorphism onto a suitably constructed sphere world. Finally, in the concluding section we sketch our understanding of the general problem and pose a number of mathematical questions whose answer would greatly advance the field of robotics. Appendix A discusses the computational complexity of the present procedure, and details of some proofs are given in Appendix B.

1.1 Problem statement. We start by defining the workspace and the obstacles.

Definition 1. Let $\beta_{j}, j \in\{0, \ldots, M\}$, be real valued analytic functions on $E^{n}$, for which zero is a regular value.

The robot workspace, $\mathscr{W}$, is a connected and compact $n$-dimensional submanifold of $E^{n}$ satisfying

$$
\stackrel{\mathscr{W}}{ } \subset\left\{q \in E^{n}: \beta_{0}(q)>0\right\} \text { and } \partial \mathscr{W} \subset\left\{q \in E^{n}: \beta_{0}(q)=0\right\} .
$$

An obstacle, $\mathscr{O}_{j}$, is the interior of a connected and compact $n$-dimensional submanifold of $E^{n}$ such that $\overline{\mathscr{O}}_{j} \subset \dot{\mathscr{W}}$, and

$$
\begin{aligned}
\mathscr{W}-\overline{\mathscr{O}}_{j} \subset\left\{q \in E^{n}: \beta_{j}(q)>0\right\} \text { and } \partial \mathscr{O}_{j} \subset\left\{q \in E^{n}: \beta_{j}(q)=0\right\}, \\
j \in\{1, \ldots, M\},
\end{aligned}
$$

satisfying

$$
\overline{\mathscr{O}}_{i} \cap \overline{\mathscr{O}}_{j}=\varnothing, \quad 1 \leq i<j \leq M
$$

The free space is

$$
\mathscr{F} \triangleq \mathscr{W}-\bigcup_{j=1}^{M} \mathscr{O}_{j} .
$$

It will prove convenient to refer to the complement of $\mathscr{W}$ in $E^{n}$ as the zeroth obstacle. Using the terminology of Thorpe [23], the free space, $\mathscr{F}$ (resulting from the removal of the obstacles from the workspace), is an analytic $n$-surface with boundary in $E^{n}$. In particular, the boundary of the $j$ th obstacle, $\partial \mathscr{O}_{j}$, is an $(n-1)$-dimensional analytic manifold, according to the Implicit Function Theorem.

Definition 2. In the special case in which the obstacles are $(n-1)$-spheres in $E^{n}$,

$$
\mathscr{O}_{0}=\{q \in E^{n}: \underbrace{\rho_{0}^{2}-\left\|q-q_{0}\right\|^{2}}_{\hat{\beta}_{0}}<0\},
$$


and

$$
\mathscr{O}_{j}=\{q \in E^{n}: \underbrace{\left\|q-q_{j}\right\|^{2}-\rho_{j}^{2}}_{\hat{\beta}_{j}}<0\}, \quad j=1, \ldots, M,
$$

the resulting free space,

$$
\mathscr{M} \triangleq\left\{q \in E^{n}: \hat{\beta}_{0}(q) \geq 0, \ldots, \hat{\beta}_{M}(q) \geq 0\right\},
$$

is an $n$-dimensional Euclidean sphere world with $M$ obstacles.

The restriction in Definition 1 to analytic submanifolds keeps our ultimate construction in this paper within the category of analytic functions on analytic manifolds. Unfortunately, even in the simplest cases-for instance, a disk robot moving amidst disk obstacles - the resulting forbidden regions possess boundaries which are not even smooth. We are adapting the results reported here to such situations where $\mathscr{F}$ is a topological, but not even a differentiable $\left(C^{(1)}\right)$ sphere world in a paper presently under preparation.

Given a pair $(\mathscr{F}, \mathscr{M})_{n, M}$ of connected and compact $n$-dimensional analytic manifolds with $M+1$ boundary components, both considered as subsets of $E^{n}$, where $\mathscr{M}$ is an $n$-dimensional sphere world with $M$ obstacles and $\mathscr{F}$ is a general $n$-dimensional free space with $M$ obstacles, let $\widetilde{\mathscr{M}}$ and $\widetilde{\mathscr{F}}$ denote some open neighborhoods about $\mathscr{M}$ and $\mathscr{F}$, respectively, in $E^{n}$. We seek a transformation $h$ from $\widetilde{F} \subset E^{n}$ into $\widetilde{\mathscr{M}} \subset E^{n}$ satisfying,

1. $h \mid \mathscr{F}$ is an analytic diffeomorphism from $\mathscr{F}$ onto $\mathscr{M}$;

2. in each space there is a distinguished interior point-the destination point $-q_{d} \in \stackrel{\circ}{\mathscr{F}}$ and $p_{d} \in \stackrel{\circ}{\mathscr{M}}$, such that $h\left(q_{d}\right)=p_{d}$.

The motivation for this problem is most simply provided by reference to our earlier paper [12]. First, some terminology. Given a map $\varphi: \mathscr{M} \rightarrow[0,1]$, adapting the terminology of M. Morse [17], we say that $\varphi$ is polar if it has a unique minimum on $\mathscr{M}$. Adapting the terminology of M. Hirsch [4], we say that $\varphi$ is admissible if it attains its maximal value (uniformly) exactly on all the boundary components-in our case, $\partial \mathscr{M}=\varphi^{-1}(1)$ and $\mathscr{M}=\varphi^{-1}[0,1)$.

Definition 3 [12, Definition 1]. Let $\mathscr{M} \subset E^{n}$ be a compact connected $n$ dimensional analytic manifold with boundary. A map $\varphi: \mathscr{M} \rightarrow[0,1]$, is a navigation function if it is

1. Analytic on $\mathscr{M}$;

2. polar on $\mathscr{M}$, with minimum at $p_{d} \in \stackrel{\circ}{\mathscr{M}}$;

3. Morse on $\mathscr{M}$;

4. admissible on $\mathscr{M}$.

It is shown in $[9,11]$ that control laws resulting from navigation functions define closed loop robotic systems whose trajectories approach the destination without intersecting obstacles, starting in an open dense set of initial states. In 
general, this is the "strongest" convergence behavior that the topology of the underlying free space allows, as we have shown in [12]. Moreover, we have shown as well that smooth navigation functions exist on any smooth manifold with boundary-hence it makes sense to attempt analytic constructions in specific cases. In particular, we have shown how to do so on any sphere world. Finally, navigation functions can be "pulled back" via diffeomorphisms, as is made precise in the following proposition.

Proposition 1.1 [12, Proposition 2.6]. Let $\varphi: \mathscr{M} \rightarrow[0,1]$ be a navigation function on $\mathscr{M}$, and let $h: \mathscr{F} \rightarrow \mathscr{M}$ be analytic. If $h$ is an analytic diffeomorphism, then

$$
\tilde{\varphi} \triangleq \varphi \circ h
$$

is a navigation function on $\mathscr{F}$.

Thus, since we already know how to construct navigation functions on any sphere world, if a suitable sphere world model, $\mathscr{M}$, and an analytic diffeomorphism, $h$, can be found, we obtain a navigation function on $\mathscr{F}$ as well.

1.2 The available information. We assume perfect information. That is, for a given star world (Definition 5 below), $\mathscr{F}$, all the "obstacle functions," $\beta_{j}$, $j \in\{0, \ldots, M\}$, as well as the obstacle center points, $q_{j}, j \in\{0, \ldots, M\}$, are known.

Let $\mathscr{S}_{j}(\varepsilon)$ denote an " $\varepsilon$-neighborhood"-a thickened neighborhood in $\mathscr{F}$ about the $j$ th obstacle, $\mathscr{O}_{j}$-defined by

$$
\mathscr{S}_{j}(\varepsilon) \triangleq\left\{q \in \mathscr{F}: 0 \leq \beta_{j}(q) \leq \varepsilon\right\}, \quad j \in\{0, \ldots, M\},
$$

where $\varepsilon$ is a positive constant.

For each obstacle, we assume the knowledge of an upper bound, $E_{j}$, on the image of the obstacle function, $\beta_{j}$, and an upper bound, $E_{d}$, on the distance from the destination point, $\gamma_{d}$, which guarantees that

$$
\begin{aligned}
\mathscr{S}_{i}\left(E_{i}\right) \cap \mathscr{S}_{j}\left(E_{j}\right)=\varnothing \text { and } \gamma_{d}^{-1}\left[0, E_{d}\right] \cap \mathscr{S}_{j}\left(E_{j}\right) & =\varnothing, \\
i, j & \in\{0, \ldots, M\}, i \neq j .
\end{aligned}
$$

That is, the " $E_{j}$-thickened" boundary components still do not intersect, nor do they overlap the destination. In consequence of the assumption that the obstacles are nonintersecting, such $\left\{E_{j}\right\}_{0}^{M}$ and $E_{d}$ exist.

Further, we will unhesitatingly make use of upper and lower bounds attained by various continuous functions on various compact sets without ever computing them explicitly. Finally, we define the notion of a "strictly star shaped obstacle" (Definition 5), and require the knowledge of a lower bound on the defining inequality (for example, $\gamma_{i}(\varepsilon)$ in Lemma 3.6, equation (19)) for each obstacle. In general, the extraction of these geometrical features from the knowledge of the obstacle functions, $\beta_{j}, j \in\{0, \ldots, M\}$, may prove to be computationally intensive. However, in [19] we presented a family of star shaped obstacle 
functions-homogeneous polynomials-for which explicit formulas for the various bounds were given.

The model sphere world, $\mathscr{M}$, is explicitly constructed from this data. That is, we determine $\left(p_{j}, \rho_{j}\right)$, the center and radius of the $j$ th sphere, according to the center and minimum "radius" (the minimal distance from $q_{j}$ to the $j$ th boundary) of the $j$ th star shaped obstacle. This in turn determines the model space "obstacle functions," $\hat{\beta}_{j}, j \in\{0, \ldots, M\}$, as well as the navigation function on $\mathscr{M}, \hat{\varphi}$, as constructed in our previous paper [12]. The transformation is then constructed in terms of the given star world and the derived model sphere world geometrical parameters.

In both spaces we explicitly assume that each obstacle contributes a distinct part of the boundary - the obstacles do not intersect each other. This assumption implies in turn that the resulting spaces are connected. Also, we require that the destination point be specified as an interior point. Once the location of the boundary components is given, the verification of the latter assumption is straightforward.

In the robotics setting, the connectedness of the free space is not a realistic assumption. Certainly, the robot initial configuration in joint space determines a specific connected component of its free space, yet this might not include the destination point. At the present, we exclude this possibility: our only test of connectedness is the application of the construction to the initial configuration. If the resulting trajectory does not arrive at the destination point, we may conclude with probability one that the destination is not in the same connected component as the initial configuration.

\section{CONSTRUCTION OF THE TRANSFORMATION}

In this section we define the star worlds, and present an explicit one-parameter family of analytic functions, each of whose members is a candidate diffeomorphism of a star world onto a particular model sphere world.

\subsection{Star worlds and their models.}

Definition 4. A set $\mathscr{S} \subset E^{n}$ with nonempty interior is star shaped (at $x_{0}$ ) if there exists a point $x_{0} \in \mathscr{S}$ such that for all $x \in \mathscr{S}$, the line segment joining $x_{0}$ and $x$ is contained in $\mathscr{S}$.

Any star shaped set is path-connected, and it can be shown [2] that any open star shaped set is homeomorphic to the open $n$-disk. According to the definition of an obstacle, if $q \in \mathscr{O}_{j}$, the $j$ th obstacle, then $\nabla \beta_{j}(q), j \in\{1, \ldots, M\}$, is directed inward with respect to the free space $\mathscr{F}$.

Definition 5. An obstacle, $\mathscr{O}_{j}$ (Definition 1), is strictly star shaped if there is a point $q_{j} \in \mathscr{O}_{j}$ such that for all $q \in \mathscr{O}_{j}$ the inward directed gradient, $\nabla \beta_{j}(q)$, satisfies

$$
\nabla \beta_{j}(q) \cdot\left(q-q_{j}\right)>0
$$


If all the obstacles in the free space, $\mathscr{F}$, are strictly star shaped, then $\mathscr{F}$ is called a star world.

The connection between the classes of strictly star shaped obstacles and star shaped sets is drawn in the following lemma.

Lemma 2.1. If $\mathscr{O}_{j}$ is a strictly star shaped obstacle, then $\mathscr{O}_{j}$ is a star shaped set $\left(\right.$ at $\left.q_{j}\right)$. Moreover, for each $q \in \partial \mathscr{O}_{j}$, the line segment joining $q_{j}$ and $q$ intersects $\partial \mathscr{O}_{j}$ only at $q$.

The proof is given in Appendix B. According to this lemma, the collection of strictly star shaped obstacles, each of whose members being compact, constitutes "almost all" possible bounded star shaped sets.

In the class of star worlds, a distinguished member is the Euclidean sphere world (Definition 2), each of whose boundary components is a scaled and translated version of the unit sphere, $S^{n-1}$.

2.2 The transformation. In this section we present a one-parameter family, each of whose members is a map induced by a specified pair $(\mathscr{F}, \mathscr{M})_{M, n}$.

Denote the omitted product, $\prod_{i=0, i \neq j}^{M} \beta_{i}$, by $\bar{\beta}_{j}$, and by $\gamma_{d}$ the (Euclidean) distance from the destination point,

$$
\gamma_{d}(q) \triangleq\left\|q-q_{d}\right\|^{2}
$$

Definition 6. The analytic switches, $\sigma_{j}, j \in\{0, \ldots, M\}$, are the real valued functions defined on $\mathscr{F}$ by

$$
\sigma_{j}(q, \lambda) \triangleq \frac{x}{x+\lambda} \circ \frac{\gamma_{d} \bar{\beta}_{j}}{\beta_{j}}=\frac{\gamma_{d} \bar{\beta}_{j}}{\gamma_{d} \bar{\beta}_{j}+\lambda \beta_{j}},
$$

where $\lambda$ is a positive constant.

Assuming that the obstacles are disjoint, the $j$ th "switch," $\sigma_{j}$, attains a uniform value of 1 on the $j$ th obstacle, vanishes on any other obstacle, and maps the interior of the free space to the open interval $(0,1)$. In the deformation scheme, sufficiently close to the $j$ th obstacle, these "switches" provide a means by which the deformation problem is reduced to the simpler problem of mapping one star shaped obstacle onto one sphere.

Recall that $\rho_{j}$ denotes the radius of the $j$ th sphere in $\mathscr{M}$.

Definition 7. The star set deforming factors, $\nu_{j}, j \in\{0, \ldots, M\}$, are the real valued functions defined on $\mathscr{F}$ by

(7) $\quad \nu_{j}(q) \triangleq \rho_{j} \frac{1+\beta_{j}(q)}{\left\|q-q_{j}\right\|}, \quad j \in\{1, \ldots, M\} \quad$ and $\quad \nu_{0}(q) \triangleq \rho_{0} \frac{1-\beta_{0}(q)}{\left\|q-q_{0}\right\|}$.

Each $\nu_{j}$ scales the ray starting at the center point of the $j$ th obstacle, $q_{j}$, through its unique intersection with the point $q \in \partial \mathscr{O}_{j}$, in such a way that $q$ is mapped to the corresponding point on the $j$ th sphere. The overall effect is 
that $\partial \mathscr{O}_{j}$ is deformed "along the rays" originating at its center point onto the corresponding sphere in model space.

Let $\widetilde{F}$ denote some open set in $E^{n}$ containing $\mathscr{F}$, the free space.

Definition 8. The star world transformation, $h_{\lambda}$, is a member of the one-parameter family of analytic maps form $\widetilde{\mathscr{F}} \subset E^{n}$ into $E^{n}$, defined by

$$
h_{\lambda}(q) \triangleq \sum_{j=0}^{M} \sigma_{j}(q, \lambda)\left[\nu_{j}(q) \cdot\left(q-q_{j}\right)+p_{j}\right]+\sigma_{d}(q, \lambda)\left[\left(q-q_{d}\right)+p_{d}\right],
$$

where $\sigma_{j}$ is the $j$ th analytic switch, $\sigma_{d}$ is defined by

$$
\sigma_{d} \triangleq 1-\sum_{j=0}^{M} \sigma_{j}
$$

and $\nu_{j}$ is the $j$ th star set deforming factor.

The "destination switch," $\sigma_{d}$, assures that $h_{\lambda}\left(q_{d}\right)=p_{d}$, that is, the star world destination point is contained in the inverse image of the sphere world destination point, a necessary condition for our method to work, since the cost function on the sphere world has a unique minimum at $p_{d}$.

Remark. This definition assumes no relation whatsoever between the location and diameter of the model space and workspace obstacles: it is only the number of boundary components in each space, $M+1$, that counts. Nevertheless, in the proof we impose two additional constraints on the model space, $\mathscr{M}$. The first assumes that the center of an obstacle in model space is identical to the corresponding center in workspace, the second ensures that the deforming factors, $\nu_{j}, j \in\{0, \ldots, M\}$, are bounded. We do not know whether these conditions are actually necessary for the desired result.

Definition 9. Given any star world, $\mathscr{F}$, the corresponding sphere world, $\mathscr{M}$, satisfies the placement condition if

$$
p_{j}=q_{j}, \quad j \in\{0, \ldots, M\}, \quad \text { and } \quad p_{d}=q_{d} .
$$

Intuitively, if $p_{d}=q_{d}$, away from the obstacles the transformation "looks like" the identity map,

$$
h_{\lambda}(q) \cong \sigma_{d}(q) \operatorname{id}(q) \cong q,
$$

provided that the parameter $\lambda$ is sufficiently large, as will be made precise later.

Recall that $\mathscr{S}_{j}(\varepsilon)$ denotes the " $\varepsilon$-neighborhood" in $\mathscr{F}$ about $\mathscr{O}_{j}$,

$$
\mathscr{S}_{j}(\varepsilon) \triangleq\left\{q \in \mathscr{F}: 0 \leq \beta_{j}(q) \leq \varepsilon\right\}, \quad j \in\{0, \ldots, M\},
$$

where $\varepsilon$ is a positive constant. 
Definition 10. Given any star world, $\mathscr{F}$, the corresponding sphere world, $\mathscr{M}$, satisfies the containment condition for $\varepsilon$ if

$$
\nu_{j}(q) \leq 1 \text { for all } q \in \mathscr{S}_{i}(\varepsilon), \quad j \in\{1, \ldots, M\},
$$

and

$$
\nu_{0}(q) \geq 1 \text { for all } q \in \mathscr{S}_{0}(\varepsilon),
$$

where $\varepsilon>0$ is small enough to guarantee that

$$
\mathscr{S}_{j}(\varepsilon) \subset \stackrel{\circ}{\mathscr{F}} \cup \mathscr{O}_{j}, \quad j \in\{0, \ldots, M\} .
$$

Remark. Evaluating $h_{\lambda}$ at a boundary point, $q \in \partial \mathscr{O}_{j}$, yields

$$
h_{\lambda}(q)=\nu_{j}(q)\left(q-q_{j}\right)+p_{j} \text {. }
$$

If the placement condition is satisfied, then the containment condition implies that

$$
\left\|h_{\lambda}(q)-q_{0}\right\| \geq\left\|q-q_{0}\right\| \text { and }\left\|h_{\lambda}(q)-q_{j}\right\| \leq\left\|q-q_{j}\right\|, \quad j \in\{1, \ldots, M\} .
$$

Geometrically this means that the $j$ th $n$-disk, $\widehat{\mathscr{O}}_{j}$, is "contained" in the $j$ th obstacle. The zeroth disk satisfies the opposite "containment" relation. Finally, given a star world, $\mathscr{F}$, we derive a model sphere world, $\mathscr{M}$, which will serve as the image space of $\mathscr{F}$ under $h_{\lambda}$.

Definition 11. Given an $n$-dimensional star world, $\mathscr{F}$, a suitable sphere world, $\mathscr{M}$, is an $n$-dimensional sphere world (Definition 2) such that $\mathscr{M}$

1. has the same number of boundary components as $\mathscr{F}$;

2. satisfies the placement condition;

3. satisfies the containment condition.

\section{PRoof that the CONSTRUCTION IS A DiffeOMORPHiSM}

In $\S 3.1$ we first characterize an analytic diffeomorphism, $h$, in terms of its Jacobian and its behavior on the boundary components, then we show that the construction of the previous section, $h_{\lambda}$, satisfies these conditions provided it has a nonsingular Jacobian on its domain. Finally, in $\S 3.2$, we prove that the Jacobian of $h_{\lambda}$ is indeed nonsingular in $\mathscr{F}$.

3.1 $h_{\lambda}$ is an analytic diffeomorphism if its Jacobian is nonsingular. In the sequel, unless otherwise stated, the spaces $\mathscr{Z}$ and $\mathscr{Y}$ denote $n$-dimensional compact connected manifolds of class $C^{(q)}$ for $q \geq 0$, with $M+1$ disjoint boundary components, which are also subsets of $E^{n}$. Denote the $j$ th boundary component of $\mathscr{X}$ and $\mathscr{Y}$ by $\partial_{j} \mathscr{X}$ and $\partial_{j} \mathscr{Y}$ respectively: each boundary component is a compact $(n-1)$-dimensional connected manifold with no boundary. Let $\widetilde{\mathscr{X}}$ denote some open neighborhood in $E^{n}$ about $\mathscr{X}$; consider the function $h \in C^{(q)}\left[\widetilde{\mathscr{X}}, E^{n}\right]$ for $q \geq 1$, we denote the set of its critical points in $\partial_{j} \mathscr{X}$ by $\mathscr{C}_{j}$ - a closed set in $\partial_{j} \mathscr{X}$. Finally, a subset of a topological space is said to be nowhere dense in the space when its closure has empty interior [7].

The following two lemmas will be used in the proposition below. 
Lemma 3.1. Let $\mathscr{B}$ be an $(n-1)$-dimensional submanifold of $\mathscr{X}$ above. Then no subset $\mathscr{C} \subset \mathscr{B}$ which is nowhere dense in $\mathscr{B}$ can disconnect $\mathscr{Z}$.

Proof. A necessary and sufficient condition that a subset $\mathscr{C} \subset \mathscr{B}$ is $(n-1)$ dimensional is that $\mathscr{C}$ contains a nonempty open subset of $\mathscr{B}[6$, Theorem 4.3]. Since $\mathscr{C}$ is nowhere dense in $\mathscr{B}$, this is not the case, and therefore

$$
\operatorname{dim}(\mathscr{C}) \leq n-2 \text {. }
$$

The result immediately follows, for no $n$-dimensional manifold can be disconnected by a subset of dimension less than $n-1$ [6, Theorem 4.4].

Lemma 3.2. Let $h: \mathscr{X} \rightarrow E^{n}$ be a continuous map such that $h(\mathscr{\mathscr { X }})$ is open in $E^{n}$. If $h$ "preserves" the boundary components,

$$
h\left(\partial_{j} \mathscr{X}\right) \subset \partial_{j} \mathscr{Y} \text { for } j \in\{0, \ldots, M\},
$$

and satisfies the condition

then $h(\mathscr{X}) \subset \mathscr{Y}$.

$$
h(\stackrel{\mathscr{X}}{)} \cap \partial \mathscr{Y}=\varnothing,
$$

Proof. The boundary of $\mathscr{Y}, \partial \mathscr{Y}$-a compact $(n-1)$-dimensional surface in $E^{n}$ consisting of $M+1$ connected components-decomposes $E^{n}$ into $M+2$ disjoint connected components whose boundary is $\partial \mathscr{Y}[13]^{1}$. This, with the connectedness of $h(\mathscr{X})$ and the fact $h(\mathscr{\mathscr { X }}) \cap \partial \mathscr{Y}=\varnothing$, imply that

$$
h\left(\stackrel { \mathscr { X } } { ) } \subset \stackrel { \circ } { \mathscr { Y } } \text { or } h \left(\stackrel{\mathscr{X}}{)} \subset E^{n}-\mathscr{Y},\right.\right.
$$

otherwise a path in $h(\mathscr{\mathscr { X }})$ passing through $\partial \mathscr{Y}$ could be found. We will show that only the first alternative is possible. Consider two cases. If $\mathscr{X}$ and $\mathscr{Y}$ have only one boundary component and $h(\stackrel{\mathscr{X}}{)}) \subset E^{n}-\mathscr{Y}$, then, since $h(\stackrel{\mathscr{X}}{)})$

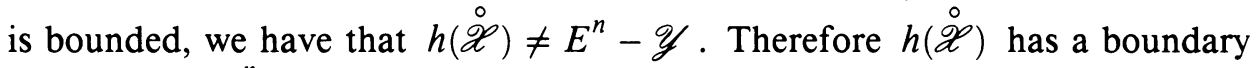
point inside $E^{n}-\mathscr{Y}$, contradicting the fact that

$$
\partial h(\stackrel{\mathscr{X}}{)} \subset h(\partial \mathscr{X}) \subset \partial \mathscr{Y},
$$

where we have used the hypotheses that $h(\stackrel{\mathscr{X}}{)})$ is open in $E^{n}$, and $h(\partial \mathscr{X}) \subset$ $\partial \mathscr{Y}$. If $\mathscr{X}$ and $\mathscr{Y}$ have more than one boundary component and $h(\stackrel{\mathscr{X}}{)} \subset$ $E^{n}-\mathscr{Y}$, then since $h$ maps $\partial_{j} \mathscr{X}$ into $\partial_{j} \mathscr{Y}$ and $E^{n}-\mathscr{Y}$ consists of at least two disjoint connected components, it must be that $h(\stackrel{\mathscr{X}}{)})$ is not connected, which is also impossible. Thus $h(\mathscr{X}) \subset \mathscr{Y}$.

We are ready to prove a sufficient condition for a map $h: \mathscr{Z} \rightarrow E^{n}$ of class $C^{(q)} q \geq 1$, to be a homeomorphism onto a given space $\mathscr{Y} \subset E^{n}$. The notation $h \in C^{(q)}[\mathscr{X}, \mathscr{Y}]$ means that $\mathscr{X}$ and $\mathscr{Y}$ have open neighborhoods in $E^{n}, \widetilde{\mathscr{X}}$ and $\widetilde{\mathscr{Y}}$ respectively, such that $h \in C^{(q)}[\widetilde{\mathscr{X}}, \widetilde{\mathscr{Y}}]$.

\footnotetext{
${ }^{1}$ The reference given applies only for $C^{(q)}$ manifolds for $q \geq 1$. According to the Alexander
} Duality Theorem [15], this fact is still true if $\partial \mathscr{Y}$ is a topological submanifold of $E^{n}$. 
Proposition 3.3. A map $h \in C^{(q)}\left[\mathscr{Z}, E^{n}\right], q \geq 1$, is a homeomorphism onto $\mathscr{Y}$ if

1. $h$ has a nonsingular Jacobian on the set $\mathscr{X}-\bigcup_{j=0}^{m} \mathscr{C}_{j}$, such that $\mathscr{C}_{j}$ is closed and nowhere dense in $\partial_{j} \mathscr{Z}$ for $j \in\{0, \ldots, M\}$;

2. $h \mid \partial_{j} \mathscr{Z}$ is a bijection into $\partial_{j} \mathscr{Y}$ for $j \in\{0, \ldots, M\}$.

3. $h$ maps some neighborhood about $\partial_{j} \mathscr{Z}$ in $\mathscr{Z}$ onto a neighborhood about $\partial_{j} \mathscr{Y}$ in $\mathscr{Y}$, for $j=0, \ldots, M$.

Remark. The possibility of critical points in $\partial \mathscr{X}$ admitted by condition 1 anticipates the future construction of navigation functions on topological sphere worlds mentioned in the introduction. For example, a paper currently in preparation treats "composite star worlds" whose obstacles arise from finite unions of star shaped sets. The resulting boundary components have "kinks"-nondifferentiable codimension 2 pieces of the smooth codimension 1 surfaceon which the Jacobian of our transformation, $h$, will become singular. For the present, our manifolds are completely smooth (actually, analytic) and our transformation, $h_{\lambda}$, has no critical points in $\partial \mathscr{X}$.

Proof. A continuous bijection on a compact space is a homeomorphism onto its image [21, Theorem 2.4], thus, in consequence of the second condition, $h \mid \partial_{j} \mathscr{Z}$ maps $\partial_{j} \mathscr{X}$ homeomorphically onto $\partial_{j} \mathscr{Y}$ : in particular, $h\left(\mathscr{C}_{j}\right)$ is nowhere dense in $\partial_{j} \mathscr{Y}$. Let $\widehat{\mathscr{X}}$ be the pre-image of the regular values of $h$. According to the first condition,

$$
\widehat{\mathscr{X}}=\mathscr{X}-\bigcup_{j=0}^{M} h^{-1}\left(h\left(\mathscr{C}_{j}\right)\right),
$$

which is open in $\mathscr{X}$. Each of the sets $h^{-1}\left(h\left(\mathscr{C}_{j}\right)\right)$ can be written as

$$
h^{-1}\left(h\left(\mathscr{C}_{j}\right)\right)=\left(h^{-1}\left(h\left(\mathscr{C}_{j}\right)\right) \cap \stackrel{0}{\mathscr{X}}\right) \cup \mathscr{C}_{j} .
$$

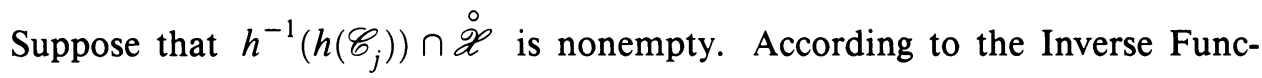
tion Theorem, $h$ is a local homeomorphism on $\mathscr{\mathscr { X }}$; therefore, since $h\left(\mathscr{C}_{j}\right)$ is

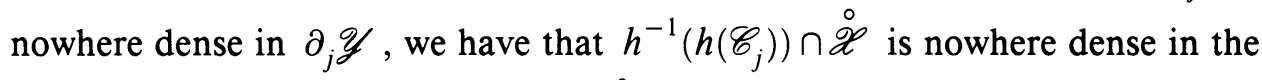
$(n-1)$-dimensional submanifold $(h \mid \grave{\mathscr{X}})^{-1}\left(\partial_{j} \mathscr{Y}\right)$. Thus, according to Lemma $3.1, \widehat{\mathscr{X}}$ is connected, and, being locally path connected, is path connected [14, Exercise 2.1].

The set $h(\widehat{\mathscr{Z}})$ - the image of a path connected set under a continuous mapis path connected, and consists of regular values of $h$. It further follows from the Inverse Function Theorem that $h$ is an open map on $\mathscr{\mathscr { X }}$ (open sets in $\mathscr{\mathscr { X }}$ are mapped to open sets in $E^{n}$ ). In particular, $h(\mathscr{\mathscr { X }})$ is open in $E^{n}$, and it follows that $\partial h(\mathscr{X}) \cap h(\stackrel{\mathscr{X}}{)}=\varnothing$. Moreover, $h(\mathscr{X})$-the image of a compact 
space under a continuous map-is compact, therefore closed in $E^{n}$, and it follows that $\partial h(\mathscr{Z}) \subset h(\mathscr{X})=h(\mathscr{X}) \cup h(\partial \mathscr{X})$. From the last two statements we conclude that

$$
h^{-1}(p) \subset \partial \mathscr{X} \text { for any point } p \in \partial h(\mathscr{X}) .
$$

By hypothesis, $h$ is injective on $\partial \mathscr{Z}$, therefore,

$$
\# h^{-1}(p)=1 \text { for all } p \in \partial h(\mathscr{X}) .
$$

We show now that

$$
h(\widehat{\mathscr{X}}) \cap \partial h(\mathscr{X}) \neq \varnothing .
$$

Supposing the contrary, it must be that

$$
\partial h(\mathscr{X}) \subset h(\mathscr{X})-h(\widehat{\mathscr{X}})=\bigcup_{j=0}^{M} h\left(\mathscr{C}_{j}\right) .
$$

Let $\mathscr{V}_{p}$ be an open connected neighborhood in $E^{n}$ about $p \in \partial h(\mathscr{X})$, and let $\mathscr{S}_{1}$ and $\mathscr{S}_{2}$ be defined by

$$
\mathscr{S}_{1} \triangleq \mathscr{V}_{p} \cap(h(\dot{\mathscr{X}})) \quad \text { and } \quad \mathscr{S}_{2} \triangleq \mathscr{V}_{p} \cap\left(E^{n}-h(\mathscr{X})\right) .
$$

$\mathscr{P}_{1}$ and $\mathscr{S}_{2}$ are disjoint open subsets of $E^{n}$. In fact,

$$
\mathscr{V}_{p}=\mathscr{S}_{1} \cup \mathscr{S}_{2} \cup\left(\mathscr{V}_{p} \cap \partial h(\mathscr{X})\right) \text {, }
$$

so it must be that the set $\mathscr{V}_{p} \cap \partial h(\mathscr{X})$ disconnects $\mathscr{V}_{p}$. But, by assumption,

$$
\mathscr{V}_{p} \cap \partial h(\mathscr{X}) \subset \mathscr{V}_{p} \cap\left(\bigcup_{j=0}^{M} h\left(\mathscr{C}_{j}\right)\right),
$$

which is nowhere dense in the $(n-1)$-manifold $\mathscr{V}_{p} \cap \partial \mathscr{Y}$. Using Lemma 3.1 again, it must be that the set $\mathscr{S}_{1} \cup \mathscr{S}_{2}$ is connected-a contradiction. We conclude that $h(\widehat{\mathscr{X}}) \cap \partial h(\mathscr{X}) \neq \varnothing$.

We shall now see that $h(\mathscr{X}) \cap \partial \mathscr{Y}=\varnothing$. Denote the $j$ th "hole" in $\mathscr{Y}$-the portion of $E^{n}-\mathscr{Y}$ bounded by $\partial_{j} \mathscr{Y}-$ by $\mathscr{O}_{j}$. Suppose to the contrary, that $h(\stackrel{\mathscr{X}}{)}) \cap \partial \mathscr{Y} \neq \varnothing$. It follows that $\mathscr{O}_{j} \cap h(\stackrel{\mathscr{X}}{)} \neq \varnothing$ for some $j \in\{0, \ldots, M\}$. Denote by $\mathscr{B}_{j}$ the neighborhood about $\partial_{j} \mathscr{Z}$ that is mapped onto a neighborhood about $\partial_{j} \mathscr{Y}$ in $\mathscr{Y}$. It must be that the set

$$
\mathscr{V} \triangleq h\left(\mathscr{B}_{j}\right) \cap h\left(\mathscr{\mathscr { X }}_{-}-\mathscr{B}_{j}\right) \neq \varnothing,
$$

since $h\left(\mathscr{B}_{j}\right) \subset \mathscr{Y}$ and $h\left(\mathscr{B}_{j}\right)$ separates $\mathscr{O}_{j}$ from the other boundary components of $\mathscr{Y}$. It follows from the definition of $\mathscr{V}$ that

$$
\# h^{-1}(p)>1 \text { for all } p \in \mathscr{V} \text {. }
$$

The fact that $h\left(\mathscr{B}_{j}\right) \subset \mathscr{Y}$ also implies that there exists a nonempty open set

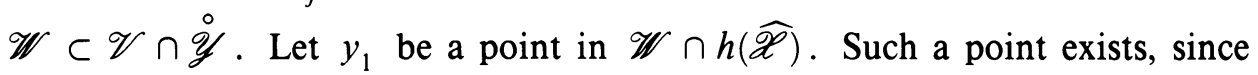


$\mathscr{W} \subset h(\grave{\mathscr{X}})$ and $h(\grave{\mathscr{X}})-h(\widehat{\mathscr{X}})$ is closed and nowhere dense in $h(\grave{\mathscr{X}})$. Let $y_{2}$ be a point in $h(\widehat{\mathscr{X}}) \cap \partial h(\mathscr{X})$. Such a point exists since $h(\widehat{\mathscr{X}}) \cap \partial h(\mathscr{X}) \neq \varnothing$. let $\alpha: I \rightarrow h(\widehat{\mathscr{X}})$ be a path such that $\alpha(0)=y_{1}$ and $\alpha(1)=y_{2}$. Such a path exists since $h(\widehat{\mathscr{X}})$ is path-connected. Moreover, we may assume that $\alpha(0,1) \subset h(\widehat{\mathscr{X}})-\partial \mathscr{Y}$, since $\alpha(0) \in \grave{\mathscr{Y}}$ and $\alpha(1) \in \partial \mathscr{Y}$. But this leads to a contradiction: $\# h^{-1}\left(y_{1}\right)>1$ and $\# h^{-1}\left(y_{2}\right)=1$, so there must be a point $y$ along $\alpha$ at which $\# h^{-1}(y)$ is not locally constant. Clearly, $y \neq y_{2}$, for $h^{-1}\left(y_{2}\right) \in \partial \mathscr{X}$, and this would imply that $h^{-1}\left(y_{2}\right)$ is a boundary point of disjoint neighborhoods in $\mathscr{X}$. Thus, $y \in h(\widehat{\mathscr{X}})-\partial \mathscr{Y}$ and it must be that $h^{-1}(y) \subset \ddot{\mathscr{X}}$. It can be shown however that this, together with the compactness of $\mathscr{X}$ and the fact that $y$ is a regular value of $h$, imply that $\# h^{-1}(p)$ is locally constant about $y[16, \mathrm{pp} .8-9]-\mathrm{a}$ contradiction.

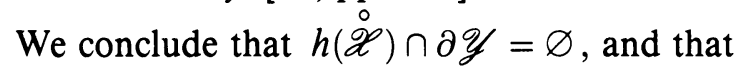

$$
\# h^{-1}(p)=1 \text { for all } p \in h(\widehat{\mathscr{X}}) \text {. }
$$

Since, in addition, $h$ is injective on $\partial \mathscr{Z}$ and $h(\partial \mathscr{Z}) \subset \partial \mathscr{Y}$, we conclude that $h$ is globally injective on $\mathscr{X}$. Moreover, according to Lemma 3.2, $h(\mathscr{X}) \subset \mathscr{Y}$.

Suppose now that $h(\mathscr{X})$ is properly contained in $\mathscr{Y}$. Since $h(\mathscr{X})$ is closed in $E^{n}$, the set $\mathscr{S} \triangleq \mathscr{Y}-h(\mathscr{X})$ is nonempty and open in $\mathscr{Y}$. Let $y_{1}$ be

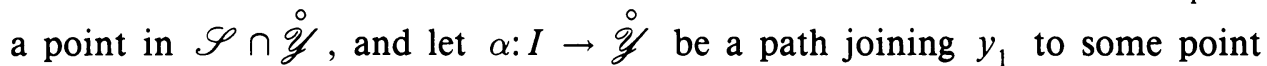

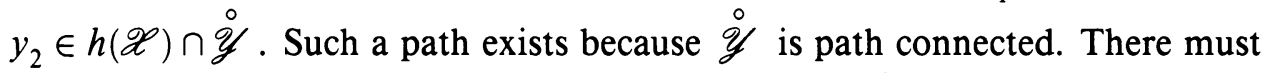
be a point along this path, $y_{3}$ say, such that $y_{3} \in \partial h(\mathscr{X})$, an impossibility, since we have shown in (12) that $\partial h(\mathscr{X}) \subset h(\partial \mathscr{X})=\partial \mathscr{Y}$.

Thus $h$ is a continuous bijection from $\mathscr{Z}$ onto $\mathscr{Y}$. Since $\mathscr{Z}$ is compact, we conclude that $h$ is a homeomorphism between $\mathscr{X}$ and $\mathscr{Y}$.

The following corollary, whose proof essentially relates the conditions of Proposition 3.3 to the structure of this section, constitutes the central contribution of the paper.

Theorem 1. For any star world possessing a valid arrangement (Definition 5), $\mathscr{F}$, there exists a suitable model sphere world (Definition 11), $\mathscr{M}$, and a positive constant $\Lambda$, such that if $\lambda \geq \Lambda$, then $h_{\lambda}: \mathscr{F} \rightarrow \mathscr{M}$, is an analytic diffeomorphism. Proof. We must show that $h_{\lambda}$ is an analytic bijection with analytic inverse. Clearly, if $\mathscr{F}$ has a valid arrangement then $h_{\lambda}$-constructed from quotients of analytic functions none of whose denominators vanishes on some open neighborhood about $\mathscr{F}$-is analytic.

In $\S 3.2$ below it is shown that for any star world, $\mathscr{F}$, with a valid arrangement, there exists a suitable model sphere world, $\mathscr{M}$, and a positive constant $\Lambda$, such that whenever $\lambda \geq \Lambda$, the Jacobian of $h_{\lambda}: \mathscr{F} \rightarrow E^{n}$ is nonsingular on $\mathscr{F}$ (as a consequence it is nonsingular on some open neighborhood in $E^{n}$ about $\mathscr{F}, \widetilde{F})$. 
We show now that $h_{\lambda}$ maps the $j$ th boundary components of $\mathscr{F}, \partial_{j} \mathscr{F}$, into the $j$ th boundary component of $\mathscr{M}, \partial_{j} \mathscr{M}$. According to Definition 1 we identify

$$
\partial_{j} \mathscr{F}=\partial \mathscr{O}_{j} \text { and } \partial_{j} \mathscr{M}=\partial \widehat{\mathscr{O}}_{j} \text { for } j \in\{0, \ldots, M\} .
$$

If $q \in \partial \mathscr{O}_{j}$ then, for a valid arrangement of the star world,

$$
\beta_{j}(q)=0 \text { and } \beta_{k}(q)>0, \quad k \in\{0, \ldots, M\}, k \neq j .
$$

Substituting in the definition of $h_{\lambda}$ yields,

$$
h_{\lambda} \mid \partial \mathscr{O}_{j}=\frac{\rho_{j}}{\left\|q-q_{j}\right\|}\left(q-q_{j}\right)+p_{j}
$$

which implies that $\left(\hat{\beta}_{j} \circ h_{\lambda}\right)(q)$, the $j$ th sphere function (Definition 2), vanishes as well. Thus,

$$
h_{\lambda}\left(\partial \mathscr{O}_{j}\right) \subset \partial \widehat{\mathscr{O}}_{j}, \quad j \in\{0, \ldots, M\} .
$$

We show now that $h_{\lambda} \mid \partial \mathscr{O}_{j}$ is injective. Suppose to the contrary, that there exist two points $q, q^{\prime} \in \partial \mathscr{O}_{j}$, such that

$$
\frac{\rho_{j}}{\left\|q-q_{j}\right\|}\left(q-q_{j}\right)+p_{j}=\frac{\rho_{j}}{\left\|q^{\prime}-q_{j}\right\|}\left(q^{\prime}-q_{j}\right)+p_{j},
$$

or

$$
\left\|q^{\prime}-q_{j}\right\|\left(q-q_{j}\right)=\left\|q-q_{j}\right\|\left(q^{\prime}-q_{j}\right) .
$$

It follows that the ray starting at $q_{j}$ through $q$ contains $q^{\prime}$ as well, an impossibility since, by hypothesis, $\mathscr{O}_{j}$ is strictly star shaped and, according to Lemma 2.1 , any such ray crosses $\partial \mathscr{O}_{j}$ exactly once.

We show now that $h_{\lambda} \mid \partial \mathscr{O}_{j}$ is surjective. If $\lambda \geq \Lambda$, there exists an open neighborhood in $E^{n}$ about $\mathscr{F}, \widetilde{\mathscr{F}}$, in which $h_{\lambda}$ has a nonsingular Jacobian. According to the Inverse Function Theorem, $h_{\lambda}$ is a local homeomorphism on $\widetilde{\mathscr{F}}$. This, together with (14) imply that $h_{\lambda} \mid \partial \mathscr{O}_{j}$ is a local homeomorphism into $\partial \widehat{\mathscr{O}}_{j}$ with their respective subspace topologies. A local homeomorphism from a compact space into a connected one is surjective. Since $\partial \mathscr{O}_{j}$ is compact and $\partial \widehat{\mathscr{O}}_{j}$ is connected, $h_{\lambda}$ maps $\partial \mathscr{O}_{j}$ onto $\partial \widehat{\mathscr{O}}_{j}$, for $j \in\{0, \ldots, M\}$.

Finally, $h_{\lambda}$ maps a neighborhood about $\partial \mathscr{O}_{i}$ in $\mathscr{F}$ onto a neighborhood about $\partial \widehat{\mathscr{O}}_{i}$ in $\mathscr{M}$. To see this, consider the ray starting at the center of $\mathscr{O}_{i}, q_{i}$ through a point $q \in \partial \mathscr{O}_{i}$, defined by

$$
r_{q}(s)=s\left(q-q_{i}\right)+q_{i} \text { for } s \geq 0 .
$$

It is shown during the proof of Proposition 3.6 that

$$
\begin{aligned}
\left.\frac{d}{d s}\right|_{s=1}\left(\hat{\beta}_{i} \circ h_{\lambda} \circ r_{q}\right)(s) & =\nabla \hat{\beta}_{i}\left(h_{\lambda}(q)\right)^{T}\left[D h_{\lambda}(q)\right]\left(q-q_{i}\right) \\
& =\left(q-q_{i}\right)^{T}\left[D h_{\lambda}(q)\right]\left(q-q_{i}\right)>0 .
\end{aligned}
$$


Since $\hat{\beta}_{i}\left(h_{\lambda}(q)\right)=0$, the last inequality implies that

$$
\left(\hat{\beta}_{i} \circ h_{\lambda}\right)\left(r_{q}(1,1+\varepsilon)\right)>0
$$

for sufficiently small $\varepsilon>0$. Since $\partial \mathscr{O}_{i}$ is compact, there exists a lower bound, $\varepsilon_{0}>0$, such that

$$
\left(\hat{\beta}_{i} \circ h_{\lambda}\right)\left(r_{q}\left(1,1+\varepsilon_{0}\right)\right)>0 \text { for all } q \in \partial \mathscr{O}_{i} .
$$

This, together with the fact that $h_{\lambda}$ is a local homeomorphism on $\stackrel{\circ}{\mathscr{F}}$, imply that $h_{\lambda}$ maps the neighborhood $\partial \mathscr{O}_{i} \times r_{q}\left[1,1+\varepsilon_{0}\right)$ onto a neighborhood about $\partial \widehat{\mathscr{O}}_{i}$ in $\mathscr{M}$.

We are now in a position to invoke Proposition 3.3, which guarantees that $h_{\lambda}$ is a homeomorphism from $\mathscr{F}$ onto $\mathscr{M}$. Since $h_{\lambda}$ is analytic and possesses a nonsingular Jacobian in $\widetilde{F}$, according to the Inverse Function Theorem [24], $h_{\lambda}$ is locally an analytic diffeomorphism ${ }^{2}$. The last two statements imply that $h_{\lambda}$ is an analytic diffeomorphism from $\mathscr{F}$ onto $\mathscr{M}$, and the proof is completed.

3.2 The Jacobian of $h_{\lambda}$ is nonsingular. In this section we compute a lower bound for the parameter $\lambda$, to guarantee that the Jacobian of $h_{\lambda}$ is nonsingular on $\mathscr{F}$. Since the technical material of this section consists of rather tedious calculations, it seems worth pausing for the intuitive motivation.

The effect of $\lambda$ becomes clear if one inspects the role of the analytic switches, $\sigma_{j}$ (Definition 6). By construction, at any point $q \in \mathscr{F}$,

$$
0 \leq \sigma_{j}(q, \lambda) \leq 1 \text { for } j \in\{0, \ldots, M\}
$$

We shall see that at a point $q$ " $\varepsilon$ away" from the $j$ th obstacle boundary i.e., a point $q \in \mathscr{F}$ at which the corresponding obstacle function $\beta_{j}(q)>\varepsilon$, for some fixed $\varepsilon>0 ; \sigma_{j}(q, \lambda)$ can be made arbitrarily small by increasing $\lambda$. Thus, if the value of $\lambda$ is sufficiently large, away from the boundary of $\mathscr{F}$ the destination switch, $\sigma_{d}=1-\sum_{j=0}^{M} \sigma_{j}$, is approximately unity, and $h_{\lambda}$ essentially looks like a perturbed vector translation,

$$
h_{\lambda}=\left[\left(q-q_{d}\right)+p_{d}\right]+\varepsilon f\left(\sigma_{0}, \ldots, \sigma_{M}\right),
$$

where $f \in C^{(\omega)}\left[\mathscr{X}, E^{n}\right]$ depends linearly on the switches $\sigma_{j}$. We shall see as well that at any such point, $q, \sigma_{j}(q, \lambda)$ can be made arbitrarily "flat"i.e., $\left\|\nabla \sigma_{j}\right\|$ can be made arbitrarily small by increasing $\lambda$. Since $D f$ depends linearly on $\sigma_{j}$ and $\nabla \sigma_{j}$ for $j \in\{0, \ldots, M\}$, it follows that away from $\partial \mathscr{F}, h_{\lambda}$ is essentially a translation of $E^{n}$, and its Jacobian is dominated by the identity matrix.

Thus the real difficulty is the "sharpening" of the analytic switches in an ever shrinking neighborhood of $\partial \mathscr{F}$ as $\lambda$ increases. Recall that the $i$ th switch was constructed to be unity on $\partial \mathscr{O}_{i}$ and to vanish at any other obstacle boundary

\footnotetext{
${ }^{2}$ It is tacitly assumed that $E^{n}$ possesses a Banach space structure.
} 
$\partial \mathscr{O}_{j}$ for $j \neq i$. By further decreasing $\varepsilon$ if necessary, consider a neighborhood about $\partial \mathscr{O}_{j}$ only, disjoint from $\mathscr{S}_{i}(\varepsilon)$ for all $i \neq j$, on which we presume to ignore the effect of the other obstacles. On $\partial \mathscr{O}_{j}$ itself, $h_{\lambda}$ becomes a simple scaling along rays of the $j$ th star onto the corresponding sphere. Since both are analytic surfaces, it is plausible (and we will prove) that $h_{\lambda} \mid \partial \mathscr{O}_{j}$ is an analytic diffeomorphism. The containment condition (Definition 10) suggests that the Jacobian of $h_{\lambda} \mid \partial \mathscr{O}_{j}$ is actually nonsingular as a map in the ambient space, that is, as a linear map from $T_{q} E^{n}$ to $T_{h_{\lambda}(q)} E^{n}$. We shall see that this is the case, and as a consequence it must be that the Jacobian of $h_{\lambda}$ is nonsingular on some open neighborhood in $E^{n}$ about $\partial \mathscr{O}_{j}$. Indeed, we will compute an upper bound for $\varepsilon$, guaranteeing that $h_{\lambda}$ has a nonsingular Jacobian on the neighborhoods $\mathscr{S}_{j}(\varepsilon)$ for $j \in\{0, \ldots, M\}$. This bound will be used in turn to compute the lower bound $\Lambda$ on the parameter $\lambda$, guaranteeing the nonsingularity of the Jacobian in the rest of $\mathscr{F}$. We may now proceed with the formal proof.

In the sequel, it is understood that any derivative of $\sigma_{j}(q, \lambda)$ is with respect to the position vector $q$, the parameter $\lambda$ being held constant.

Consider the set $\mathscr{S}_{j}(\varepsilon)$-the "thickened" $j$ th boundary component-defined in equation (2) as

$$
\mathscr{S}_{j}(\varepsilon)=\left\{q \in \mathscr{F}: 0 \leq \beta_{j}(q) \leq \varepsilon\right\},
$$

with the constraint $\varepsilon \leq E_{i}$. That is, $\varepsilon$ is small enough to guarantee that the sets $\mathscr{S}_{j}(\varepsilon), j \in\{0, \ldots, M\}$, are disjoint and do not overlap the destination point.

We distinguish in the star world the set "away from the obstacles,"

$$
\mathscr{A}(\varepsilon) \triangleq\left\{q \in \mathscr{F}: \beta_{0}(q)>\varepsilon, \ldots, \beta_{M}(q)>\varepsilon\right\},
$$

and denote its complement in $\mathscr{F}$ by

$$
\mathscr{A}^{c}(\varepsilon) \triangleq \mathscr{F}-\mathscr{A}(\varepsilon)=\bigsqcup_{j=0}^{M} \mathscr{S}_{j}(\varepsilon),
$$

a disjoint union.

We are ready to prove the nonsingularity of the Jacobian on the set "away" from the obstacles, $\mathscr{A}(\varepsilon)$.

In the Appendix we prove in Lemma B.4 and Lemma B.5 that for any $\varepsilon>0$ and any $\delta>0, \sigma_{j}(q, \lambda)$ and $\left\|\nabla \sigma_{j}(q, \lambda)\right\|$ can be made to be smaller than $\delta$ on the set "away" from the $j$ th obstacle, $\mathscr{F}-\mathscr{S}_{j}(\varepsilon)$, by choosing

$$
\lambda \geq N_{0 j}(\varepsilon, \delta) \text { and } \lambda \geq N_{1 j}(\varepsilon, \delta),
$$

respectively, where $N_{0 j}(\varepsilon, \delta), N_{1 j}(\varepsilon, \delta)$ are positive constants. As a consequence, it is possible to guarantee that the Jacobian of $h_{\lambda}$ is nonsingular on $\mathscr{A}(\varepsilon)$, a statement which is made precise as follows.

Lemma 3.4. Given a valid arrangement of the star world, $\mathscr{F}$, for any $\varepsilon>0$ there exists a positive constant $\Lambda_{0}(\varepsilon)$ such that if $\lambda \geq \Lambda_{0}$ then $D h_{\lambda}$ is nonsingular on $\mathscr{A}(\varepsilon)$. 
Proof. Under the placement condition (Definition 9), the Jacobian of $h_{\lambda}$ is shown in Lemma B.2 to be

$$
D h_{\lambda}(q)=\sum_{j=0}^{M}\left\{\sigma_{j} \nu_{j} I+\sigma_{j}\left(q-q_{j}\right) \nabla \nu_{j}^{T}+\left(\nu_{j}-1\right)\left(q-q_{j}\right) \nabla \sigma_{j}^{T}\right\}+\sigma_{d} I .
$$

Let $\hat{x}$ be any vector based at $q \in \mathscr{A}(\varepsilon)$, that is, $\hat{x} \in T_{q} \mathscr{A}(\varepsilon)$. Evaluating the Jacobian along $\hat{x}$ yields,

$$
\begin{aligned}
{[D h(q)] \hat{x}=} & \left(\sum_{j=0}^{M} \sigma_{j} \nu_{j}+\sigma_{d}\right) \hat{x} \\
& +\overbrace{\left[\sum_{j=0}^{M}\left\{\sigma_{j}\left(q-q_{j}\right) \nabla \nu_{j}^{T}+\left(\nu_{j}-1\right)\left(q-\sigma_{j}\right) \nabla \sigma_{j}^{T}\right\}\right]}^{\left.\hat{x} \sigma_{j}\right)},
\end{aligned}
$$

where $w\left(\sigma_{j}, \nabla \sigma_{j}\right)$ is a shorthand notation for $w\left(\sigma_{j}, \nabla \sigma_{j}\right), j \in\{0, \ldots, M\}$. We will use the positive magnitude of $\sigma_{d}$ to dominate $w$. First, note that the latter may be bounded from above in magnitude by

$$
\left\|w\left(\sigma_{j}, \nabla \sigma_{j}\right)\right\|<\frac{1}{2} \text {. }
$$

For, choosing

$$
\lambda>\max _{j \in\{0, \ldots, M\}}\left\{N_{0 j}(\varepsilon, \delta), N_{1 j}(\varepsilon, \delta)\right\},
$$

it follows from Lemma B.4 and Lemma B.5 that $\sigma_{j}(q, \lambda)$ and $\left\|\nabla \sigma_{j}(q, \lambda)\right\|$ are bounded from above by $\delta>0$; hence,

$$
\begin{aligned}
\left\|w\left(\sigma_{j}, \nabla \sigma_{j}\right)\right\| & \leq \sum_{j=0}^{M}\left\|q-q_{j}\right\|\left(\sigma_{j}\left\|\nabla \nu_{j}\right\|+\left|\nu_{j}-1\right|\left\|\nabla \sigma_{j}\right\|\right) \\
& <\delta \sum_{j=0}^{M}\left\|q-q_{j}\right\|\left(\left\|\nabla \nu_{j}\right\|+\left|\nu_{j}-1\right|\right) .
\end{aligned}
$$

A sufficient condition on $\delta$ for the desired inequality is thus

$$
\delta \leq \frac{1}{2 \max _{\mathscr{F}}\left\{\sum_{j=0}^{M}\left\|q-q_{j}\right\|\left(\left\|\nabla \nu_{j}(q)\right\|+\left|\nu_{j}(q)-1\right|\right)\right\}} \triangleq \delta_{0}^{\prime} .
$$

Note that $\nu_{j}$ is analytic on the compact set $\mathscr{F}$, and in consequence both $\nu_{j}$ and $\left\|\nabla \nu_{j}\right\|$ are bounded, thus, there is no problem with the definition of $\delta_{0}^{\prime}$.

On the other hand, note that $\sigma_{d}$ may be bounded from below by $\frac{1}{2}$, for, again according to Lemma B.4 and Lemma B.5, by choosing $\lambda$ sufficiently large, we may impose on the switches the condition

$$
\sigma_{j}(q, \lambda) \leq \frac{1}{2} \frac{1}{1+M} \text { for all } q \in \mathscr{A}(\varepsilon), j \in\{0, \ldots, M\},
$$


which implies that

$$
\sigma_{d}=1-\sum_{j=0}^{M} \sigma_{j} \geq \frac{1}{2}
$$

Finally, choosing

$$
\delta_{0} \triangleq \min \left\{\delta_{0}^{\prime}, \frac{1}{2} \frac{1}{1+M}\right\}
$$

the desired $\Lambda_{0}$ is

$$
\Lambda_{0}(\varepsilon) \triangleq \max _{j \in\{0, \ldots, M\}}\left\{N_{0 j}\left(\varepsilon, \delta_{0}\right), N_{1 j}\left(\varepsilon, \delta_{0}\right)\right\},
$$

and the result follows.

We turn now to the set $\mathscr{A}^{c}(\varepsilon)$.

First we have to establish that for a given star world, $\mathscr{F}$, a suitable model sphere world, $\mathscr{M}$, can be found. In particular, $\mathscr{M}$ must satisfy the containment condition (Definition 10). The following Lemma specifies upper bounds on the radii of the spheres (a lower bound for the 0th sphere) in $\mathscr{M}$, guaranteeing that the containment condition is satisfied in the "thickened obstacles" neighborhoods $\mathscr{S}_{j}\left(E_{j}\right)$.

Recall that $\rho_{j}$ denotes the radius of the $j$ th sphere in $\mathscr{M}$.

Lemma 3.5. For each $j \in\{0, \ldots, M\}$, there exists a positive real number $R_{j}\left(E_{j}\right)$ such that if $\rho_{j} \leq R_{j}$, then

$$
\nu_{j}(q) \leq 1 \text { for all } q \in \mathscr{S}_{j}\left(E_{j}\right)
$$

and if $\rho_{0} \geq R_{0}$, then

$$
\nu_{0}(q) \geq 1 \text { for all } q \in \mathscr{S}_{0}\left(E_{0}\right) .
$$

Proof. According to its definition in equation (7),

if

$$
\nu_{j}(q)=\rho_{j} \frac{1+\beta_{j}(q)}{\left\|q-q_{j}\right\|} \leq 1,
$$

which is implied by the condition

$$
\rho_{j} \leq \frac{\left\|q-q_{j}\right\|}{1+\beta_{j}(q)}
$$

$$
\rho_{j} \leq \frac{\min _{q \in \partial \mathscr{O}_{j}}\left\{\left\|q-q_{j}\right\|\right\}}{1+E_{j}} \triangleq R_{j}\left(E_{j}\right), \quad j \in\{1, \ldots, M\},
$$

since $0 \leq \beta_{j} \leq E_{j}$, and

$$
\min _{q \in \partial \mathscr{O}_{j}}\left\{\left\|q-q_{j}\right\|\right\} \leq \min _{q \in \mathscr{S}_{j}\left(E_{j}\right)}\left\{\left\|q-q_{j}\right\|\right\} .
$$

In the case of $\nu_{0}$, using a similar argument, the condition

$$
\rho_{0} \geq \frac{\max _{q \in \mathcal{O}_{0}}\left\{\left\|q-q_{0}\right\|\right\}}{1-E_{0}} \triangleq R_{0}\left(E_{0}\right),
$$

is sufficient to guarantee that $\nu_{0} \geq 1$. 
$D h_{\lambda}(q)$ maps $T_{q} \mathscr{F}$ into $T_{h_{\lambda}(q)} h_{\lambda}(\mathscr{F})$, both of which are $n$-dimensional vector spaces, and therefore isomorphic to $E^{n}$. Since the domains of $h_{\lambda}$-the star world, $\mathscr{F}$, is an $n$-dimensional submanifold of $E^{n}$, we will not distinguish points in the tangent space from points in the original base spaces.

Let $\left(q-q_{i}\right)$ be a vector based at $q \in E^{n}$. Consider the tangent space to $\mathscr{F}$ at $q, T_{q} \mathscr{F}$, as the orthogonal direct sum

$$
T_{q} \mathscr{F}=\left\langle q-q_{i}\right\rangle \oplus\left\langle q-q_{i}\right\rangle^{\perp},
$$

where $\langle\cdot\rangle$ denotes the "span of," and $(\cdot)^{\perp}$ denotes the "orthogonal complement of." Each vector $x \in T_{q} \mathscr{F}$ can be uniquely written as

$$
x=x_{1}+x_{2} \text { such that } x_{1} \in\left\langle q-q_{i}\right\rangle \text { and } x_{2} \in\left\langle q-q_{i}\right\rangle^{\perp} .
$$

Given a nonzero vector $v$, denote its associated unit vector, $v /\|v\|$, by $\hat{v}$.

We are ready to prove the existence of a neighborhood in $\mathscr{F}$ about $\partial \mathscr{F}$, $\mathscr{A}^{c}(\varepsilon)$, in which $D h_{\lambda}$ is nonsingular.

Proposition 3.6. Given a valid arrangement of the star world, $\mathscr{F}$, and a suitable sphere world, $\mathscr{M}$, for each $j \in\{0, \ldots, M\}$ there exist positive constants, $\varepsilon_{j}$ and $\Lambda_{j}$, such that for all $\varepsilon \leq \varepsilon_{j}$ and $\lambda \geq \Lambda_{j}$, the Jacobian of $h_{\lambda}$ is nonsingular on the set

$$
\mathscr{S}_{j}(\varepsilon)=\left\{q \in \mathscr{F}: 0 \leq \beta_{j}(q) \leq \varepsilon\right\} .
$$

Proof. In a valid arrangement of the star world, each obstacle is strictly star shaped (Definition 5), that is,

$$
\zeta_{j}(q) \triangleq\left(q-q_{j}\right) \cdot \nabla \beta_{j}>0 \quad \text { for all } q \in \partial \mathscr{O}_{j},
$$

and it follows from the continuity of $\zeta_{j}$ that this condition holds in some neighborhood in $\mathscr{F}$ about $\partial \mathscr{O}_{j}$, for instance,

$$
\mathscr{S}_{j}\left(\varepsilon_{j 0}\right)=\left\{q \in \mathscr{F}: 0 \leq \beta_{j}(q) \leq \varepsilon_{j 0}\right\},
$$

where $\varepsilon_{j 0} \leq E_{j}$.

We partition the unit sphere in $T_{q} \mathscr{F}$, denoted by $S_{q}^{n}$, into two disjoint "cones,"

$$
\mathscr{C}_{q} \triangleq\left\{x \in S_{q}^{n}: \frac{\left\|x_{1}\right\|}{\left\|x_{2}\right\|}>\frac{\sqrt{2}}{\widehat{\nabla \beta_{j}} \cdot\left(q-q_{j}\right)}\right\} \quad \text { and }\left(\mathscr{C}_{q}\right)^{c}=S_{q}^{n}-\mathscr{C}_{q} \text {. }
$$

In the Appendix, before the statement of Lemma B.8, we motivate this particular decomposition.

First suppose that $\hat{x} \in \mathscr{C}_{q}$. In Lemma B.8 we show the existence of two constants, $\varepsilon_{j 1} \leq \varepsilon_{j 0}$ and $\Lambda_{j 1}$, such that for all $\varepsilon \leq \varepsilon_{j 1}$ and $q \in \mathscr{S}_{j}(\varepsilon)$, if $\lambda \geq \Lambda_{j 1}$, then

$$
\hat{x}^{T}\left[D h_{\lambda}(q)\right] \hat{x}>0 \text { for all } \hat{x} \in \mathscr{C}_{q},
$$

in particular, $\left[D h_{\lambda}(q)\right] \hat{x} \neq 0$. 
Consider the complementary case: at each tangent space, the test directions belong to the "cone" $S_{q}^{n}-\mathscr{C}_{q}$, in which

$$
\left\|x_{2}\right\| \geq \frac{1}{\sqrt{2}}\left[\left(q \widehat{-q}_{j}\right) \cdot \widehat{\nabla \beta}_{j}\right]\left\|x_{1}\right\| .
$$

In Lemma B.9 we show the existence of two constants, $\varepsilon_{j 2} \leq \varepsilon_{j 0}$ and $\Lambda_{j 2}$, such that for all $\varepsilon \leq \varepsilon_{j 2}$ and $q \in \mathscr{S}_{j}(\varepsilon)$, if $\lambda \geq \Lambda_{j 2}$, then

$$
x_{2}^{T}\left[D h_{\lambda}(q)\right] \hat{x}>0 \text { for all } \hat{x} \in S_{q}^{n}-\mathscr{C}_{q},
$$

where $\hat{x}=x_{1}+x_{2}$ as above. In particular, $\left[D h_{\lambda}(q)\right] \hat{x} \neq 0$.

By definition, whenever $\varepsilon_{1} \leq \varepsilon_{2}$ we have that $\mathscr{S}_{j}\left(\varepsilon_{1}\right) \subset \mathscr{S}_{j}\left(\varepsilon_{2}\right)$, and it follows that any bound on $\varepsilon$ or $\lambda$ in the set $\mathscr{S}_{j}\left(\varepsilon_{2}\right)$ a fortiori applies to the smaller set $\mathscr{S}_{j}\left(\varepsilon_{1}\right)$. Therefore, letting

$$
\varepsilon_{j} \triangleq \min \left\{\varepsilon_{j 1}, \varepsilon_{j 2}\right\} \quad \text { and } \quad \Lambda_{j} \triangleq \max \left\{\Lambda_{j 1}, \Lambda_{j 2}\right\}
$$

completes the proof.

Since we have considered the entirety of $\mathscr{F}$, the above results are summarized in the following corollary.

Corollary 3.7. Given a valid arrangement of $\mathscr{F}$, there exist a suitable sphere world, $\mathscr{M}$ and a positive constant, $\Lambda$, such that if $\lambda \geq \Lambda$, then the Jacobian of $h_{\lambda}: \mathscr{F} \rightarrow \mathscr{M}$ is nonsingular on $\mathscr{F}$.

Proof. First we choose a suitable sphere world, $\mathscr{M}$. To do so, we fix the centers of the spheres and the model destination point in accordance with the placement condition (Definition 9),

$$
p_{j}=q_{j}, \quad j \in\{0, \ldots, M\} \text { and } p_{d}=q_{d},
$$

where $q_{j}$ is the center of the $j$ th star, and $q_{d}$ the destination point. Then, using Lemma 3.5, we fix the sphere radii such that,

$$
\nu_{0}(q) \geq 1 \text { for all } q \in \mathscr{S}_{0}\left(E_{0}\right)
$$

and,

$$
\nu_{j}(q) \leq 1 \text { for } j \in\{1, \ldots, M\} \text { and for all } q \in \mathscr{S}_{j}\left(E_{j}\right),
$$

which is the containment condition.

Using Proposition 3.6, we choose a constant,

$$
\varepsilon^{*} \triangleq \min _{j \in\{0, \ldots, M\}}\left\{\varepsilon_{j}\right\},
$$

designating a neighborhood in $\mathscr{F}$ about $\partial \mathscr{F}, \mathscr{A}^{c}\left(\varepsilon^{*}\right)$, in which $D h_{\lambda}$ is nonsingular, whenever the parameter $\lambda$ satisfies

$$
\lambda \geq \max _{j \in\{0, \ldots, M\}}\left\{\Lambda_{j}\right\} \triangleq \Lambda_{1} .
$$


According to Lemma 3.4 , for any $\varepsilon^{*}>0$, if the parameter $\lambda$ satisfies $\lambda \geq$ $\Lambda_{0}\left(\varepsilon^{*}\right)$, then $D h_{\lambda}$ is nonsingular in the set $\mathscr{A}\left(\varepsilon^{*}\right)$. Thus, letting

$$
\Lambda \triangleq \max \left\{\Lambda_{0}\left(\varepsilon^{*}\right), \Lambda_{1}\right\},
$$

completes the proof.

\section{CONClusion}

We have constructed an algebraic analytic diffeomorphism between two classes of analytic submanifolds of $E^{n}$. In Appendix A we show that the computational complexity of this transformation, beyond that introduced by the boundary functions themselves, is proportional to $M n^{2}+M^{2}$, where $M$ is the number of obstacles and $n$ the dimension of $\mathscr{F}$. A numerical example is provided in Figure 1: a planar $(n=2)$ star world, $\mathscr{F}$, with five internal obstacles, and its corresponding model sphere world, $\mathscr{M}$. We plot the level lines of a navigation function on $\mathscr{F}, \varphi$, as well as those of the corresponding navigation function on $\mathscr{M}, \hat{\varphi}$. The parameter in $\hat{\varphi}$ is chosen sufficiently high to eliminate spurious local minima in $\mathscr{M}$. The destination point in both spaces is chosen arbitrarily at the origin, and the level lines vary regularly between zero (at the destination point), and one (on all the boundary components). It can be seen that the pulled back navigation function, $\varphi=\hat{\varphi} \circ h_{\lambda}$, for an appropriately chosen $\lambda$, introduces no additional critical points. Thus there is a unique minimum at the destination point, and one saddle point near each (internal) star obstacle. As we have shown in [12], one cannot do better than this using smooth vector fields which are transverse to the boundary of $\mathscr{F}$.

These results should be of interest to mathematicians since we construct analytic diffeomorphisms in closed form using algebraic operations: our functions do not arise from the one-parameter group of any obvious dynamical system. From the point of view of engineering applications, our efforts comprise the first exact robot navigation algorithms using the "artificial potential field" methodology [8]. Moreover, as indicated by a number of remarks throughout the body of this paper, current work in progress gives us reason to hope that extensions of these ideas will yield exact navigation algorithms on a family of spaces which is dense in the topological equivalence class of our original model-the Euclidean sphere world [12]. However, this model is itself unrealistic. Since we do not possess, at present, a classification theory for the topology of freespace arising from general navigation problems, a significant gulf still remains between our present understanding and a practicable methodology for guiding robots reliably in cluttered environments.

Acknowledgement. We would like to thank Professor M. Hirsch who originally suggested the utility of diffeomorphisms in this context, and Professor W. Massey for his kind unstinting tutorial efforts on our behalf. 

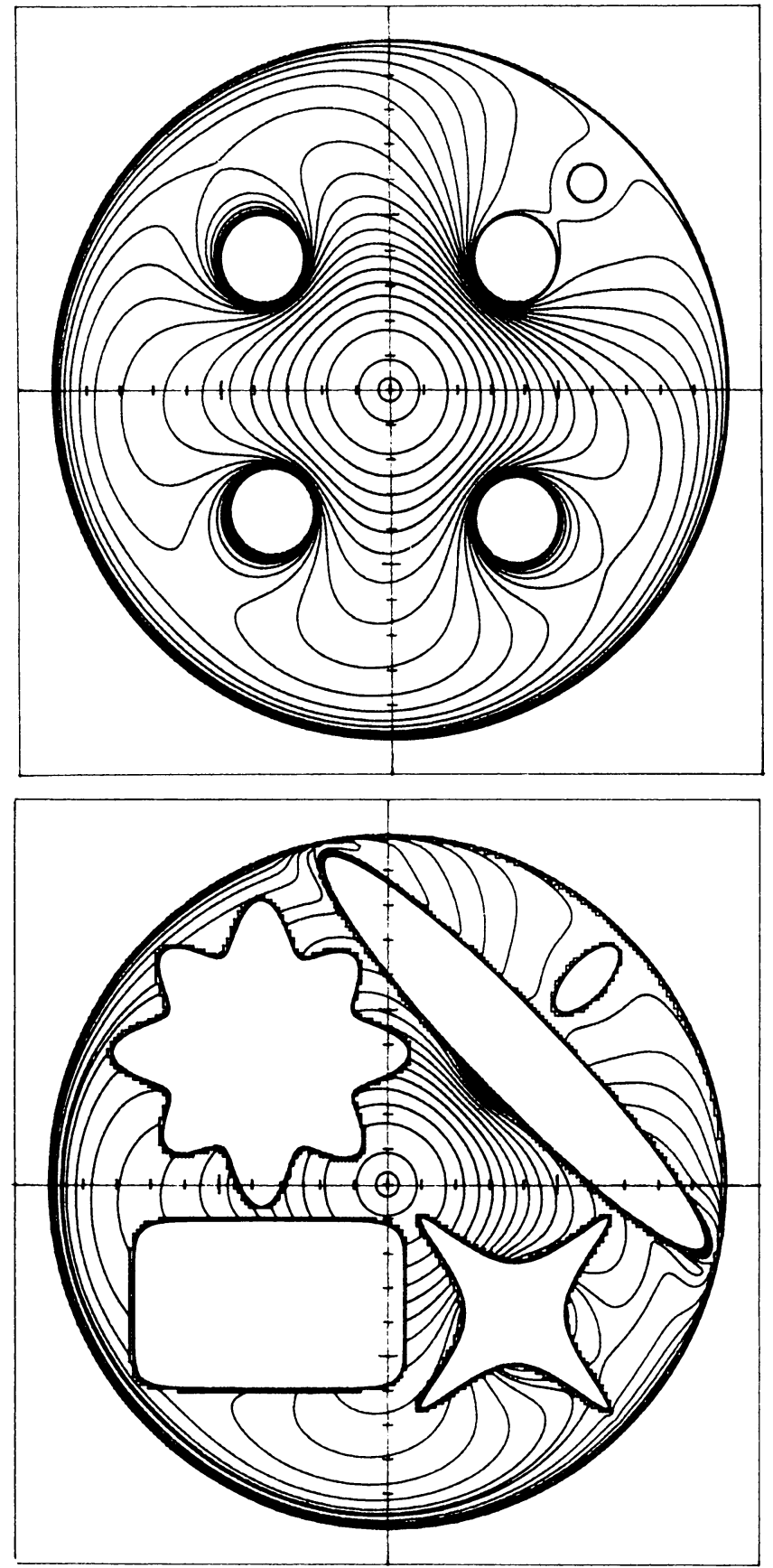

FIGURE 1

Planar $(n=2)$ star world (bottom), $\mathscr{F}$, and its corresponding model sphere world (top), for the case $M=5$ internal obstacles. The resulting navigation function on $\mathscr{F}$ possesses a unique minimum-zero-at the destination point, chosen arbitrarily as the origin, a saddle point near each (internal) obstacle, and is exactly one on $\partial \mathscr{F}$. 


\section{A. Counting the floAting POINT Operations}

The computation involved has two parts. First, when presented with the data (specified in $\S 1.2$ ), describing a star world with a valid arrangement, $\mathscr{F}$, we construct a navigation function on $\mathscr{F}, \varphi=\hat{\varphi} \circ h_{\lambda}$, by choosing a suitable sphere world, $\mathscr{M}$, constructing $\hat{\varphi}$-the navigation function on $\mathscr{M}$, and choosing the parameter $\lambda$ in $h_{\lambda}$. The computational complexity of this part is analyzed in $\S A .1$. Second, the controller has to compute $\nabla \varphi$,

$$
\nabla \varphi=\nabla\left(\hat{\varphi} \circ h_{\lambda}\right)=\left[D h_{\lambda}\right]^{T} \nabla \hat{\varphi}\left(h_{\lambda}\right),
$$

and we analyze the computational complexity of this term in $\S A .2$.

A.1 The computation of the parameters in $\varphi$. The count of the floating point operations will be given in terms of $M$-the number of obstacles, and $n$-the dimension of the ambient Euclidean space. We defined the obstacle functions, $\beta_{i}$, as real valued analytic functions describing strictly star shaped obstacles (Definition 5). In order to speak meaningfully about the number of floating point operations, we restrict the obstacle functions to the class of positive definite homogeneous polynomials of degree $k \in \mathbf{N}$. In [19] we show that each member of this class-which is essentially all the polynomials which satisfy the properties of a norm except, possibly, the triangular inequality-describes a strictly star shaped obstacle. It is important to note that this class serves only as an example. Although such functions generate a great variety of star shapes, we currently do not know whether this class is rich enough to represent "almost all" the star shaped obstacles.

Under this restriction, it turns out that in order to compute the parameters in $h_{\lambda}$ for a star world, $\mathscr{F}$, the following data suffices,

1. for each obstacle, $\mathscr{O}_{j}$, its center point, $q_{j}$, the obstacle function, $\beta_{j}$, and its (homogeneous) degree $k_{j}$;

2. for each obstacle, $\mathscr{O}_{j}$, a radius, loosely denoted by

$$
\min _{q \in \partial \mathscr{O}_{j}}\left\{\left\|q-q_{j}\right\|\right\}, \quad j \in\{0, \ldots, M\},
$$

such that the sphere with this radius, when centered at $q_{j}$, is contained in $\mathscr{O}_{j}$ (contains $\mathscr{O}_{0}$ for the zeroth obstacle);

3. a destination point, $q_{d} \in \stackrel{\circ}{\mathscr{F}}$;

4. the upper bounds on the obstacle functions, $\left\{E_{j}\right\}_{0}^{M}$, and on the distance to $q_{d}, E_{d}$, which were specified in $\S 1.2$.

In [19] we provide practical formulas that render all the relevant terms in the computation of the parameter of $h_{\lambda}$ in terms of this data. The steps in constructing $h_{\lambda}$ from the data were summarized in Corollary 3.7, and are repeated here,

1. choose a suitable sphere world for $\left\{E_{j}\right\}_{j=0}^{M}$ and $E_{d}$, and compute its parameter, $k$;

2. compute $\varepsilon^{*}$ from the data; 
3. find $\Lambda\left(\varepsilon^{*}\right)$, a lower bound on the parameter $\lambda$ in $h_{\lambda}$. In the first step-choosing a suitable sphere world-we start by fixing the sphere obstacle centers and the destination point according to the placement condition (Definition 11), and no computation is involved. Next, we compute an upper bound on the radius of the $i$ th sphere (lower bound for the zeroth sphere), $\rho_{i}$, using Lemma 3.5. This involves, roughly, one operation per obstacle. As for the parameter $k$ of the navigation function on the corresponding sphere world, $\hat{\varphi}$, we refer the reader to [11], in which we showed that this computation takes no more than $10 M^{2} n$ operations. Therefore this step takes no more than $10 M^{2} n+5 M$ operations.

In the second step, $\varepsilon^{*}$ is derived from $\varepsilon_{i 1}$ and $\varepsilon_{i 2}$ for $i \in\{0, \ldots, M\}$, specified in Lemma B.8 and Lemma B.9, respectively. The computation of $\varepsilon_{i 1}$, defined in equations (38) and (42), is dominated by the terms

$$
\max _{\mathscr{F}}\left\{\beta_{j}\right\}, \quad \max _{\mathscr{F}}\left\{\left\|\nabla \beta_{j}\right\|\right\}, \quad \max _{\mathscr{F}}\left\{\left\|q-q_{j}\right\|\right\} \quad \text { for } j \in\{0, \ldots, M\} .
$$

We provide in [19] formulas for computing $\max _{\mathscr{F}}\left\{\beta_{j}\right\}$ and $\max _{\mathscr{F}}\left\{\left\|\nabla \beta_{j}\right\|\right\}$ in, roughly, one operation. The third term satisfies

$$
\max _{\mathscr{F}}\left\{\left\|q-q_{j}\right\|\right\} \leq \rho_{0},
$$

by the containment condition, and therefore does not require any computation. The computation of $\varepsilon_{i 2}$, defined in equation (50), takes, roughly, one operation. We conclude that $\varepsilon^{*}$ can be computed in less than $10 M$ operations.

In the third step-computing $\Lambda\left(\varepsilon^{*}\right)$, a lower bound for $\lambda$-we have to compute $\Lambda_{0}\left(\varepsilon^{*}\right)$ and $\Lambda_{j}$ for $j \in\{0, \ldots, M\}$, specified in Lemma 3.4 and Proposition 3.6, respectively. $\Lambda_{0}$ is derived from the terms $N_{0 j}\left(\varepsilon^{*}, \delta\right)$ and $N_{1 j}\left(\varepsilon^{*}, \delta\right)$ for $j \in\{0, \ldots, M\}$. First we have to compute $\delta$, which according to its definition in equation (15), involves a summation of the terms

$$
\max _{\mathscr{F}}\left\{\left\|q-q_{j}\right\|\left|\nu_{j}-1\right|\right\} \quad \text { and } \max _{\mathscr{F}}\left\{\left\|q-q_{j}\right\|\left\|\nabla \nu_{j}\right\|\right\}, \quad j \in\{0, \ldots, M\} \text {. }
$$

Expanding $\nu_{j}$ (equation (7)) and $\nabla \nu_{j}$ (equation (28)) yields

$$
\begin{aligned}
\left\|q-q_{j}\right\|\left|\nu_{j}-1\right| & =\rho_{j}\left|1+\beta_{j}-\left\|q-q_{j}\right\|\right| \\
& \leq \rho_{j}\left(1+\max _{\mathscr{G}}\left\{\beta_{j}\right\}+\max _{\mathscr{F}}\left\{\left\|q-q_{j}\right\|\right\}\right),
\end{aligned}
$$

and

$$
\begin{aligned}
\left\|q-q_{j}\right\|\left\|\nabla \nu_{j}\right\| & =\rho_{j}\left\|\nabla \beta_{j}-\frac{1+\beta_{j}}{\left\|q-q_{j}\right\|}\left(\widehat{q-q_{j}}\right)\right\| \\
& \leq \rho_{j}\left(\max _{\mathscr{F}}\left\{\left\|\nabla \beta_{j}\right\|\right\}+\frac{1+\max _{\mathscr{F}}\left\{\beta_{j}\right\}}{\min _{\partial \mathscr{O}_{j}}\left\{\left\|q-q_{j}\right\|\right\}}\right) .
\end{aligned}
$$

Since the minimal radius of $\mathscr{O}_{j}, \min _{\partial \mathcal{O}_{j}}\left\{\left\|q-q_{j}\right\|\right\}$, is part of the data, and all the other terms were already computed in (22), it takes less than $5 M$ additional 
operations to compute $\delta$. We turn now to the computation of $N_{0 j}$ and $N_{1 j}$. According to Lemma B.4 and Lemma B.5, their computation involves the terms

$$
\max _{\mathscr{F}}\left\{\beta_{j}\right\} \text { and } \max _{\mathscr{F}}\left\{\left\|\nabla \beta_{j}\right\|\right\} \text { for } j \in\{0, \ldots, M\} \text {, }
$$

which were already computed; and it takes less than $5 M$ additional operations to compute $N_{0 j}$ and $N_{1 j}$ for the $j$ th obstacle. Thus, $\Lambda_{0}$ can be computed in less than $10 M+10 M^{2}$ operations.

According to Proposition 3.6, $\Lambda_{j}$ is derived from $\Lambda_{j 1}$ and $\Lambda_{j 2}$ for $j \in$ $\{0, \ldots, M\}$, defined in Lemma B.8 and Lemma B.9, respectively. Using equations (41) and (43) for $\Lambda_{j 1}$, and equations (49) and (51) for $\Lambda_{j 2}$, both involve a composition of the terms listed in equations (23) and (24) above, which were already computed. Thus it takes less than $10 \mathrm{M}$ additional operations to compute $\Lambda_{j 1}$ and $\Lambda_{j 2}$ for the $j$ th obstacle, and it follows that the computation of $\Lambda\left(\varepsilon^{*}\right)$ takes less than $10 M+15 M^{2}$ operations.

Summing up, the total number of floating point operations required is bounded by

$$
10 M^{2} n+15 M^{2}
$$

where $M$ is the number of internal obstacles and $n$ the dimension of $\mathscr{F}$.

Remark. The dimension of the space, $n$, appears only in the computation of the sphere world parameter, $k$. This is a consequence of the assumption we have made about the allowable obstacle functions-homogeneous polynomials, which enabled us to give explicit scalar bounds on all the required terms.

A.2 The computation of $\nabla \varphi$. Using equation (21), the computation of $\nabla \varphi(q)$ involves the following steps,

1. compute $p \triangleq h_{\lambda}(q)$;

2. compute $\nabla \hat{\varphi}(p)$;

3. compute $D h_{\lambda}(q)$;

4. multiply the matrix $D h_{\lambda}(q)^{T}$ by the vector $\nabla \hat{\varphi}(p)$.

Denote the number of floating point operations required to compute the $i$ th obstacle function, $\beta_{i}$, and its gradient, $\nabla \beta_{i}$, by $\#\left(\beta_{i}\right)$ and $\#\left(\nabla \beta_{i}\right)$. The number of operations required will be given in terms of $\#\left(\beta_{i}\right), \#\left(\nabla \beta_{i}\right), M$ and $n$.

From its definition (Definition 8), the computation of $h_{\lambda}$ involves the summation of $M+1$ terms, each of the form,

$$
\sigma_{i}(q)\left[\nu_{i}(q) \cdot\left(q-q_{i}\right)+p_{i}\right]
$$

According to their definition (equation (6)), the computation of the analytic switches, $\left\{\sigma_{j}\right\}_{j=0}^{M}$, involves the product of $\left\{\beta_{j}\right\}_{j=0}^{M}$ and $\gamma_{d}$. Therefore it takes less than $5 M+\sum_{j=0}^{M} \#\left(\beta_{j}\right)$ operations to compute the analytic switches. Next, according to its definition (equation (7)), the computation of the $i$ th star deforming factor, $\nu_{i}$, involves, roughly, the quotient of $\beta_{i}$ with $\left\|q-q_{i}\right\|$. Therefore it takes less than $5 M n$ additional operations to compute $\left\{\nu_{j}\right\}_{j=0}^{M}$. We 
conclude that it takes no more than $10 M n+\sum_{j=0}^{M} \#\left(\beta_{j}\right)$ operations to compute $h_{\lambda}(q)$.

In the second step-computing $\nabla \hat{\varphi}(p)$-we refer the reader to [11], in which we showed that it takes no more than $10\left(M^{2}+M n\right)$ operations to compute $\nabla \hat{\varphi}(p)$.

Using equation (27) for $D h_{\lambda}$, the computation in the third step involves the summation of $M+1$ terms, each of the form

$$
\sigma_{i} \nu_{i} I+\sigma_{i}\left(q-q_{i}\right) \nabla \nu_{i}^{T}+\left(\nu_{i}-1\right)\left(q-q_{i}\right) \nabla \sigma_{i}^{T} .
$$

Rewriting $\nabla \nu_{i}$ from equation (28),

$$
\nabla \nu_{i}(q)=\frac{\nu_{i}}{\left\|q-q_{i}\right\|}\left(\frac{\left\|q-q_{i}\right\|}{1+\beta_{i}} \nabla \beta_{i}-\left(q-q_{i}\right)\right) .
$$

Since $\nu_{i}$ and $\left\|q-q_{i}\right\|$ were already computed, it takes $\#\left(\nabla \beta_{i}\right)+10 n$ additional operations to compute $\nabla \nu_{i}$. Rewriting $\nabla \sigma_{i}$ (equation 29),

$$
\begin{aligned}
\nabla \sigma_{i} & =\frac{\lambda}{\left(\gamma_{d} \bar{\beta}_{i}+\lambda \beta_{i}\right)^{2}}\left(\beta_{i} \nabla\left(\gamma_{d} \bar{\beta}_{i}\right)-\gamma_{d} \bar{\beta}_{i} \nabla \beta_{i}\right) \\
& =\frac{\lambda}{\left(\gamma_{d} \bar{\beta}_{i}+\lambda \beta_{i}\right)^{2}}\left(\sum_{j=0, j \neq i}^{M} \gamma_{d} \bar{\beta}_{j} \nabla \beta_{j}+\beta \nabla \gamma_{d}-\gamma_{d} \bar{\beta}_{i} \nabla \beta_{i}\right) .
\end{aligned}
$$

Since $\beta_{j}$ and $\nabla \beta_{j}$ were already computed, it takes $10 \mathrm{Mn}$ additional operations to compute $\nabla \sigma_{i}$. Thus, the computation of (26) takes no more than $5 n^{2}$ additional operations, and we conclude that the computation of $D h_{\lambda}(q)$ is bounded by $10\left(M n^{2}+\sum_{J=0}^{M} \#\left(\nabla \beta_{j}\right)\right)$ additional operations.

Summing up (the fourth step takes $5 n^{2}$ operations), we conclude that it takes no more than

$$
10\left\{M n^{2}+M^{2}+\sum_{j=0}^{M}\left\{\#\left(\beta_{j}\right)+\#\left(\nabla \beta_{j}\right)\right\}\right\}
$$

operations to compute $\nabla \varphi(q)$.

Remark. If we instantiate the obstacle functions within the class of polynomials of degree $k$ or less, then, in general, each such polynomial consists of $k$ homogeneous polynomials, each of which can have no more than $\left(\begin{array}{c}n+k-1 \\ k\end{array}\right)$ terms, therefore,

$$
\#\left(\beta_{i}\right)=k\left(\begin{array}{c}
n+k-1 \\
k
\end{array}\right) \text { and } \#\left(\nabla \beta_{i}\right)=n(k-2)\left(\begin{array}{c}
n+k-2 \\
k-1
\end{array}\right) .
$$

Thus, if we relate $k$ to the "geometric complexity," and $n$ to the number of degrees of freedom of the underlying kinematic chain, then, assuming that $k \geq n$, the computation involved is proportional to $k^{n}$, i.e. exponential in the number of degrees of freedom and polynomial in the geometric complexity. 


\section{B. Computational details}

This section contains various lemmas which are used in $\S 3$. We begin with a lemma of a topological nature, which is used in $\S 3.1$. All the other lemmas are used to prove the nonsingularity of $D h_{\lambda}$ in $\S 3.2$.

Let $\widetilde{\mathscr{X}}, \widetilde{\mathscr{Y}}$ denote $n$-dimensional manifolds without boundary, and let $\mathscr{X} \subset$ $\widetilde{\mathscr{X}}, \mathscr{Y} \subset \widetilde{\mathscr{Y}}$ be $n$-dimensional compact submanifolds with $M+1$ boundary components. Suppose that $h \in C^{(\omega)}[\widetilde{\mathscr{Z}}, \widetilde{\mathscr{Y}}]$, if the Jacobian of $h$ is nonsingular on $\widetilde{\mathscr{Z}}$, then the Inverse Function Theorem guarantees that $h$ is locally a smooth diffeomorphism: in particular, $h$ is a local homeomorphism.

Lemma B.1. If $h: \widetilde{\mathscr{X}} \rightarrow \widetilde{Y}$ is a local homeomorphism, such that

$$
h(\partial \mathscr{X}) \subset \partial \mathscr{Y} \text { and } h(\mathscr{\mathscr { X }}) \subset \stackrel{\mathscr{Y}}{ } \text {, }
$$

then $h \mid \mathscr{X}: \mathscr{Z} \rightarrow \mathscr{Y}$ is a local homeomorphism in the subspace topology.

Proof. It suffices to show that each point $q \in \partial \mathscr{Z}$ has an open neighborhood $\mathscr{U}$ in the subspace topology of $\mathscr{Z}$ such that $h(\mathscr{U})$ is open in $\mathscr{Y}$ and $h$ maps $\mathscr{U}$ homeomorphically onto $h(\mathscr{U})$.

Let $\tilde{\mathscr{U}}$ be an open $n$-disc in $\widetilde{\mathscr{X}}$ about $q \in \partial \mathscr{X}$, on which $h$ is homeomorphic. By assumption, the set $\widetilde{\mathscr{V}} \triangleq h(\tilde{\mathscr{U}})$ is an open $n$-disc in $\widetilde{\mathscr{Y}}$ about $p \triangleq h(q) \in \partial \mathscr{Y}$.

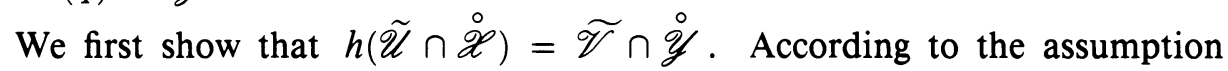
$h(\stackrel{\mathscr{X}}{)} \subset \stackrel{\circ}{\mathrm{Y}}$,

$$
h(\tilde{\mathscr{U}} \cap \stackrel{\mathscr{X}}{)} \subset h(\tilde{\mathscr{U}}) \cap h(\stackrel{\mathscr{X}}{)} \subset \widetilde{\mathscr{V}} \cap \stackrel{\mathscr{Y}}{ },
$$

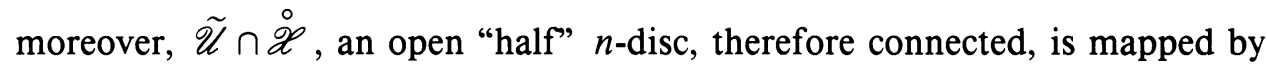

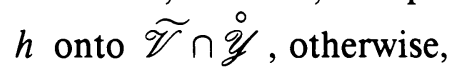

$$
(h \mid \widetilde{\mathscr{U}})^{-1}(\widetilde{\mathscr{V}} \cap \stackrel{\mathscr{Y}}{)}) \subset\left(\tilde{\mathscr{U}} \cap \stackrel{\mathscr{X}}{)} \cup\left(\tilde{\mathscr{U}} \cap \mathscr{X}^{c}\right),\right.
$$

is a disconnected set. To avoid contradiction (with the fact that the image of a connected set under a continuous map is connected), it must be that

$$
(h \mid \widetilde{\mathscr{U}})^{-1}(\widetilde{\mathscr{V}} \cap \stackrel{\mathscr{Y}}{)} \subset \tilde{\mathscr{U}} \cap \stackrel{\mathscr{X}}{ },
$$

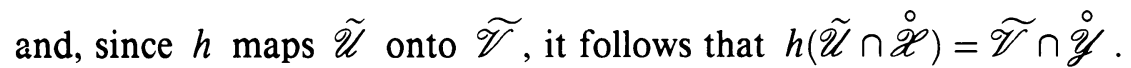

It is also true that $h(\widetilde{\mathscr{U}} \cap \partial \mathscr{X})=\widetilde{\mathscr{V}} \cap \partial \mathscr{Y}$, for,

$$
h(\tilde{\mathscr{U}} \cap \partial \mathscr{X}) \subset h(\tilde{\mathscr{U}}) \cap h(\partial \mathscr{X}) \subset \widetilde{\mathscr{V}} \cap \partial \mathscr{Y} .
$$

On the other hand,

$$
h(\tilde{\mathscr{U}} \cap \mathscr{X})=h(\tilde{\mathscr{U}} \cap \stackrel{\mathscr{X}}{)} \cup h(\tilde{\mathscr{U}} \cap \partial \mathscr{X})=(\widetilde{\mathscr{V}} \cap \dot{\mathscr{Y}}) \cup h(\tilde{\mathscr{U}} \cap \partial \mathscr{X}) .
$$


The set $h(\tilde{\mathscr{U}} \cap \mathscr{X})$ is closed in $\widetilde{\mathscr{V}}$, since $\tilde{\mathscr{U}} \cap \mathscr{X}$ is closed in $\tilde{\mathscr{U}}$, therefore $h(\tilde{\mathscr{U}} \cap \partial \mathscr{X})$ contains the set of boundary points of $\widetilde{\mathscr{V}} \cap \mathscr{\mathscr { Y }}$ in $\widetilde{\mathscr{V}}$, which is exactly $\widetilde{\mathscr{V}} \cap \partial \mathscr{Y}$. Thus,

$$
\widetilde{\mathscr{V}} \cap \partial \mathscr{Y} \subset h(\tilde{\mathscr{U}} \cap \partial \mathscr{X}),
$$

and it follows that $h(\tilde{\mathscr{U}} \cap \partial \mathscr{X})=\tilde{\mathscr{V}} \cap \partial \mathscr{Y}$. We conclude that

$$
h(\tilde{\mathscr{U}} \cap \mathscr{X})=(\widetilde{\mathscr{V}} \cap \dot{\mathscr{Y}}) \cup(\widetilde{\mathscr{V}} \cap \partial \mathscr{Y})=\widetilde{\mathscr{V}} \cap \mathscr{Y},
$$

and the result follows.

Remark. Using local homology groups, one can show [15] that if $h: \mathscr{Z} \rightarrow \mathscr{Y}$ is a local homeomorphism between $n$-dimensional manifolds with boundary, then

$$
h(\stackrel{\mathscr{X}}{)}) \stackrel{\circ}{\mathscr{Y}} \text { and } h(\partial \mathscr{X}) \subset \partial \mathscr{Y} .
$$

B.1 Some general computations. First, we give a proof of Lemma 2.1.

Lemma 2.1. If $\mathscr{O}_{j}$ is a strictly star shaped obstacle (Definition 5), then $\mathscr{O}_{j}$ is a star shaped set $\left(\right.$ at $\left.q_{j}\right)$. Moreover, for each $q \in \partial \mathscr{O}_{j}$, the line segment joining $q_{j}$ and $q$ intersects $\partial \mathscr{O}_{j}$ only at $q$.

Proof. According to Definition 5, $q_{j} \in \mathscr{O}_{j}$. We have to show that for each point $q \in \mathscr{O}_{j}$, the line segment joining $q_{j}$ and $q$ is contained in $\mathscr{O}_{j}$. Let $r:[0,1] \rightarrow E^{n}$ be a continuous parametrization of this line segment, defined by

$$
r(\lambda) \triangleq q_{j}+\lambda\left(q-q_{j}\right) .
$$

Suppose to the contrary, that there exists $\lambda_{1} \in(0,1)$ such that $q^{\prime} \triangleq r\left(\lambda_{1}\right) \notin \mathscr{O}_{j}$. We lose no generality by assuming that

$$
\lambda_{1}=\inf \left\{\lambda>0: r(\lambda) \notin \mathscr{O}_{j}\right\},
$$

which implies that $q^{\prime} \in \partial \mathscr{O}_{j}$, and therefore $\left(\beta_{j} \circ r\right)\left(\lambda_{1}\right)=0$. According to equation (4) in the definition of a strictly star shaped obstacle,

$$
\nabla \beta_{j}\left(q^{\prime}\right) \cdot\left(q^{\prime}-q_{j}\right)>0,
$$

and it follows by continuity argument that there exists $\varepsilon>0$ such that

$$
\left(\frac{d}{d \lambda} \beta_{j} \circ r\right)\left(\lambda_{1}-\varepsilon, \lambda_{1}+\varepsilon\right)>0 \text {. }
$$

Now suppose that $r$ does not cross $\partial \mathscr{O}_{j}$ into $\left(\mathscr{O}_{j}\right)^{c}$ at $\lambda_{1}$, in particular, there exists $\lambda^{*} \in\left(\lambda_{1}, \lambda_{1}+\varepsilon\right)$ such that

$$
\left(\beta_{j} \circ r\right)\left(\lambda^{*}\right) \leq 0 .
$$

According to the Mean Value Theorem,

$$
\left(\beta_{j} \circ r\right)\left(\lambda^{*}\right)-\left(\beta_{j} \circ r\right)\left(\lambda_{1}\right)=\left(\lambda^{*}-\lambda_{1}\right) \frac{d}{d \lambda}\left(\beta_{j} \circ r\right)(s)>0,
$$


for some $s \in\left(\lambda_{1}, \lambda^{*}\right)$, which implies that $\left(\beta_{j} \circ r\right)\left(\lambda^{*}\right)>0$, a contradiction. Therefore, $\left(\beta_{j} \circ r\right)\left(\lambda_{1}, \lambda_{1}+\varepsilon\right)>0$, and it follows that

$$
r\left(\lambda_{1}, \lambda_{1}+\varepsilon\right) \subset\left(\mathscr{O}_{i}\right)^{c} .
$$

We show now that $r$ cannot cross $\partial \mathscr{O}_{j}$ again. Suppose to the contrary, that there exists $\lambda_{2}>\lambda_{1}$ such that $q^{\prime \prime} \triangleq r\left(\lambda_{2}\right) \in \partial \mathscr{O}_{j}$, that is, $\left(\beta_{j} \circ r\right)\left(\lambda_{2}\right)=0$. We lose no generality by assuming that

$$
\lambda_{2}=\inf \left\{\lambda>\lambda_{1}: r(\lambda) \in \partial \mathscr{O}_{j}\right\} .
$$

It follows that

$$
r\left(\lambda_{1}, \lambda_{2}\right) \subset\left(\mathscr{O}_{j}\right)^{c}
$$

which in turn implies that

$$
\left(\beta_{j} \circ r\right)\left(\left(\lambda_{2}-\varepsilon, \lambda_{2}\right)\right)>0
$$

for some $\varepsilon>0$. By assumption,

$$
\nabla \beta_{j}\left(q^{\prime \prime}\right) \cdot\left(q^{\prime \prime}-q_{j}\right)>0,
$$

and it follows by an argument similar to the one given above that

$$
\left(\beta_{j} \circ r\right)\left(\lambda_{2}\right)>\left(\beta_{j} \circ r\right)\left(\lambda_{2}-\frac{1}{2} \varepsilon\right)>0,
$$

a contradiction. Thus the assumption that the line segment crosses $\partial \mathscr{O}_{j}$ at $q^{\prime}$ leads to a contradiction with the fact that its endpoint, $q$, is in $\mathscr{O}_{j}$. It must be that this line segment is contained in $\mathscr{O}_{j}$, and, crosses $\partial \mathscr{O}_{j}$ only when its endpoint is in $\partial \mathscr{O}_{j}$.

The following technical lemma computes the Jacobian of $h_{\lambda}$.

Lemma B.2. Assuming the placement condition (Definition 9), the Jacobian of the star world deformation, $h_{\lambda}$, is

$$
D h(q)=\sum_{j=0}^{M}\left\{\sigma_{j} \nu_{j} I+\sigma_{j}\left(q-q_{j}\right) \nabla \nu_{j}^{T}+\left(\nu_{j}-1\right)\left(q-q_{j}\right) \nabla \sigma_{j}^{T}\right\}+\sigma_{d} I .
$$

Proof. Using equation (8), the Jacobian of $h_{\lambda}$ is

$$
\begin{aligned}
D h= & \sum_{j=0}^{M}\left\{\sigma_{j} \nu_{j} I+\sigma_{j}\left(q-q_{j}\right) \nabla \nu_{j}^{T}+\left(\nu_{j}\left(q-q_{j}\right)+p_{j}\right) \nabla \sigma_{j}^{T}\right\} \\
& +\sigma_{d} I+\left(\left(q-q_{d}\right)+p_{d}\right) \nabla \sigma_{d}^{T} .
\end{aligned}
$$

As $\sigma_{d}$ was defined to be $\sigma_{d}=1-\sum_{j=0}^{M} \sigma_{j}$, substituting $\nabla \sigma_{d}$ obtains,

$$
\begin{aligned}
D h=\sum_{j=0}^{M}\left\{\sigma_{j} \nu_{j} I\right. & +\sigma_{j}\left(q-q_{j}\right) \nabla \nu_{j}^{T} \\
& \left.+\left[\left(\nu_{j}\left(q-q_{j}\right)+p_{j}\right)-\left(\left(q-q_{d}\right)+p_{d}\right)\right] \nabla \sigma_{j}^{T}\right\}+\sigma_{d} I,
\end{aligned}
$$


assuming the placement condition, that is, $p_{j}=q_{j}, j \in\{0, \ldots, M\}$, and $p_{d}=$ $q_{d}$, obtains

$$
D h=\sum_{j=0}^{M}\left\{\sigma_{j} \nu_{j} I+\sigma_{j}\left(q-q_{j}\right) \nabla \nu_{j}^{T}+\left(\nu_{j}-1\right)\left(q-q_{j}\right) \nabla \sigma_{j}^{T}\right\}+\sigma_{d} I .
$$

Denote a unit magnitude vector, $v$ say, by $\hat{v}$. The following lemma gives a formula for the gradient of the star set deforming factors.

Lemma B.3. The gradient of the star set deforming factor (Definition 8), is

$$
\nabla \nu_{j}(q)=\nu_{j}\left(\frac{1}{1+\beta_{j}} \nabla \beta_{j}-\frac{1}{\left\|q-q_{j}\right\|}\left(\widehat{q-q_{j}}\right)\right),
$$

for $j \in\{1, \ldots, M\}$, and

$$
\nabla \nu_{0}(q)=\nu_{0}\left(-\frac{1}{1-\beta_{0}} \nabla \beta_{0}-\frac{1}{\left\|q-q_{0}\right\|}\left(q-q_{0}\right)\right),
$$

when $j=0$.

Proof. The $j$ th analytic switch was defined in Definition 7 to be

$$
\nu_{j}(q)=\rho_{j} \frac{1+\beta_{j}(q)}{\left\|q-q_{j}\right\|}, \quad j \in\{1, \ldots, M\} \quad \text { and } \quad \nu_{0}(q)=\rho_{0} \frac{1-\beta_{0}(q)}{\left\|q-q_{0}\right\|},
$$

thus,

$$
\begin{aligned}
\nabla \nu_{j} & =\frac{\rho_{j}}{\left\|q-q_{j}\right\|^{2}}\left(\left\|q-q_{j}\right\| \nabla \beta_{j}-\frac{1+\beta_{j}}{\left\|q-q_{j}\right\|}\left(q-q_{j}\right)\right) \\
& =\frac{\rho_{j}\left(1+\beta_{j}\right)}{\left\|q-q_{j}\right\|^{2}}\left(\frac{\left\|q-q_{j}\right\|}{1+\beta_{j}} \nabla \beta_{j}-\frac{1}{\left\|q-q_{j}\right\|}\left(q-q_{j}\right)\right) \\
& =\nu_{j}\left(\frac{1}{1+\beta_{j}} \nabla \beta_{j}-\frac{1}{\left\|q-q_{j}\right\|}\left(q-q_{j}\right)\right) .
\end{aligned}
$$

The case where $j=0$ yields a similar result.

We show now that if the parameter $\lambda$ is large enough, the $j$ th switch, $\sigma_{j}$, and its normed gradient, $\left\|\nabla \sigma_{j}\right\|$, can be made arbitrarily small on $\mathscr{F}-\mathscr{S}_{i}(\varepsilon)$. Lemma B.4. For any $\varepsilon>0, \delta>0$, and $j \in\{0, \ldots, M\}$, there exists a positive real number $N_{0 j}(\varepsilon, \delta)$ such that if $\lambda \geq N_{0 j}$ then

Proof.

$$
\sigma_{j}(q, \lambda) \leq \delta \text { for all } q \in \mathscr{F}-\mathscr{S}_{j}(\varepsilon) \text {. }
$$

$$
\sigma_{j}=\frac{\gamma_{d} \bar{\beta}_{j}}{\gamma_{d} \bar{\beta}_{j}+\lambda \beta_{j}} \leq \frac{\gamma_{d} \bar{\beta}_{j}}{\lambda \beta_{j}}
$$

for the term on the right to be less than $\delta, \lambda$ must satisfy,

$$
\lambda \geq \frac{1}{\delta} \frac{\gamma_{d} \bar{\beta}_{j}}{\beta_{j}}
$$


Since $\beta_{j}>\varepsilon$ in $\mathscr{F}-\mathscr{S}_{j}(\varepsilon)$, a sufficient condition on $\lambda$ is

$$
\lambda \geq \frac{1}{\delta \varepsilon} \max _{\mathscr{F}}\left\{\gamma_{d} \bar{\beta}_{j}\right\} \triangleq N_{0 j}(\varepsilon, \delta) .
$$

Lemma B.5. For any $\varepsilon>0, \delta>0$, and $j \in\{0, \ldots, M\}$ there exists a positive real number $N_{1 j}(\varepsilon, \delta)$ such that if $\lambda \geq N_{1 j}$ then

$$
\left\|\nabla \sigma_{j}(q, \lambda)\right\| \leq \delta \text { for all } q \in \mathscr{F}-\mathscr{S}_{j}(\varepsilon) .
$$

Proof.

$$
\nabla \sigma_{j}=\frac{\lambda}{\left(\gamma_{d} \bar{\beta}_{j}+\lambda \beta_{j}\right)^{2}}\left(\beta_{j} \nabla\left(\gamma_{d} \bar{\beta}_{j}\right)-\gamma_{d} \bar{\beta}_{j} \nabla \beta_{j}\right),
$$

which implies that

$$
\left\|\nabla \sigma_{j}\right\| \leq \frac{\lambda}{\left(\gamma_{d} \bar{\beta}_{j}+\lambda \beta_{j}\right)^{2}}\left(\beta_{j}\left\|\nabla\left(\gamma_{d} \bar{\beta}_{j}\right)\right\|+\gamma_{d} \bar{\beta}_{j}\left\|\nabla \beta_{j}\right\|\right),
$$

and since $\lambda \beta_{j} /\left(\gamma_{d} \bar{\beta}_{j}+\lambda \beta_{j}\right) \leq 1$,

$$
\begin{aligned}
\left\|\nabla \sigma_{j}\right\| & \leq \frac{1}{\gamma_{d} \bar{\beta}_{j}+\lambda \beta_{j}}\left\|\nabla\left(\gamma_{d} \bar{\beta}_{j}\right)\right\|+\frac{1}{\beta_{j}} \frac{\gamma_{d} \bar{\beta}_{j}}{\gamma_{j}+\lambda \beta_{j}}\left\|\nabla \beta_{j}\right\| \\
& \leq \frac{1}{\lambda \beta_{j}}\left\|\nabla\left(\gamma_{d} \bar{\beta}_{j}\right)\right\|+\frac{\gamma_{d} \bar{\beta}_{j}}{\lambda \beta_{j}^{2}}\left\|\nabla \beta_{j}\right\| .
\end{aligned}
$$

The assumption that $\beta_{j}>\varepsilon$ in $\mathscr{F}-\mathscr{S}_{j}(\varepsilon)$ yields as a sufficient condition,

$$
\lambda \geq \frac{1}{\delta}\left(\frac{1}{\varepsilon} \max _{\mathscr{F}}\left\{\left\|\nabla\left(\gamma_{d} \bar{\beta}_{j}\right)\right\|\right\}+\frac{1}{\varepsilon^{2}} \max _{\mathscr{F}}\left\{\gamma_{d} \bar{\beta}_{j}\left\|\nabla \beta_{j}\right\|\right\}\right) \triangleq N_{1 j}(\varepsilon, \delta) .
$$

B.2. The set "near" the $i$ th obstacle. The results of this section are concerned with $\mathscr{S}_{i}(\varepsilon)$ - the set "near" the $i$ th obstacle.

The following lemmas are used in Lemma B. 8 that follows. The intuitive motivation for Lemma B. 6 below is best provided by the consideration of the "symmetric part" of the matrix $\nabla \beta_{i}\left(q-q_{i}\right)^{T}$, defined by

$$
\left[\nabla \beta_{i}\left(q-q_{i}\right)^{T}\right]_{s} \triangleq \frac{1}{2}\left(\nabla \beta_{i}\left(q-q_{i}\right)^{T}+\left(q-q_{i}\right) \nabla \beta_{i}^{T}\right) .
$$

The quadratic form associated with $\left[\nabla \beta_{i}\left(q-q_{i}\right)^{T}\right]_{s}$ is positive on the subspace $\left\langle q-q_{i}\right\rangle \subset T_{q}^{\mathscr{F}}$,

$$
\left(q-q_{i}\right)^{T}\left[\nabla \beta_{i}\left(q-q_{i}\right)^{T}\right]_{s}\left(q-q_{i}\right)=\left\|q-q_{i}\right\|^{2}\left(q-q_{i}\right) \cdot \nabla \beta_{i}>0,
$$

at any point $q \in \mathscr{F}$ satisfying $\left(q-q_{i}\right) \cdot \nabla \beta_{i}>0$. Under this assumption, it follows that there exists an open neighborhood in $T_{q} \mathscr{F}$ about $\left\langle q-q_{i}\right\rangle$ on which this quadratic form is positive. In particular, the following lemma designates a "cone" about $\left\langle q-q_{i}\right\rangle$ contained in this neighborhood. 
Lemma B.6. Let $q$ be a point in $\mathscr{F}$ at which $\left(q-q_{i}\right) \cdot \nabla \beta_{i}>0$. If $x \in T-q \mathscr{F}$ satisfies

$$
\left\|x_{1}\right\| \geq \frac{\sqrt{2}}{\left({\left.\widehat{q-q_{i}}\right) \cdot \widehat{\nabla \beta}}_{i}\right.}\left\|x_{2}\right\|
$$

where $x=x_{1}+x_{2}$ such that $x_{1} \in\left\langle q-q_{i}\right\rangle$ and $x_{2} \in\left\langle q-q_{i}\right\rangle^{\perp}$, then

$$
x^{T}\left[\nabla \beta_{i}\left(q-q_{i}\right)^{T}\right] x>0 .
$$

Moreover, for any unit vector $\hat{x} \in T_{q} \mathscr{F}$ satisfying (30),

$$
\left\|x_{1}\right\|^{2} \geq \frac{2}{3} \text { and }\left|\nabla \beta_{i} \cdot \hat{x}\right| \geq \frac{1}{6}\left(\widehat{q-q_{i}}\right) \cdot \nabla \beta_{i} .
$$

Proof. Using the identity $x_{1} \cdot \nabla \beta_{i}=\left(x_{1} \cdot\left(\widehat{q-q_{i}}\right)\right)\left(\left(\widehat{q-q_{i}}\right) \cdot \nabla \beta_{i}\right)$,

$$
\begin{aligned}
x^{T}[\nabla & \left.\beta_{i}\left(q-q_{i}\right)^{T}\right] x=\left[\left(x_{1}+x_{2}\right) \cdot \nabla \beta_{i}\right]\left(\left(q-q_{i}\right) \cdot x_{1}\right) \\
& =\left[\left(x_{1} \cdot\left(q-q_{i}\right)\right)\left(\left(q-q_{i}\right) \cdot \nabla \beta_{i}\right)+x_{2} \cdot \nabla \beta_{i}\right]\left(\left(q-q_{i}\right) \cdot x_{1}\right) \\
& \geq\left\|q-q_{i}\right\|\left\{\left(\left(q-q_{i}\right) \cdot \nabla \beta_{i}\right)\left|x_{1} \cdot\left(q \widehat{-q_{i}}\right)\right|^{2}-\left\|x_{2}\right\|\left\|\nabla \beta_{i}\right\|\left|x_{1} \cdot\left(\widehat{q-q_{i}}\right)\right|\right\} .
\end{aligned}
$$

Since $x_{1} \in\left\langle q-q_{i}\right\rangle$,

$$
x^{T}\left[\nabla \beta_{i}\left(q-q_{i}\right)^{T}\right] x \geq\left\|q-q_{i}\right\|\left\|x_{1}\right\|\left\{\left(\left(\widehat{q-q_{i}}\right) \cdot \nabla \beta_{i}\right)\left\|x_{1}\right\|-\left\|x_{2}\right\|\left\|\nabla \beta_{i}\right\|\right\}>0,
$$

whenever

$$
\left\|x_{1}\right\|>\frac{1}{\left(\widehat{q-q}_{i}\right) \cdot \widehat{\nabla \beta_{i}}}\left\|x_{2}\right\|,
$$

which is implied by (30).

We turn now to the second assertion. First we compute a lower bound on $\left\|x_{1}\right\|$. Using (30),

$$
1=\left\|x_{1}\right\|^{2}+\left\|x_{2}\right\|^{2} \leq\left\|x_{1}\right\|^{2}\left(1+\frac{1}{2}\left[\left(q \widehat{-q_{i}}\right) \cdot \widehat{\nabla \beta_{i}}\right]^{2}\right) \leq \frac{3}{2}\left\|x_{1}\right\|^{2},
$$

thus, $\left\|x_{1}\right\|^{2} \geq \frac{2}{3}$. Using (30) again,

$$
\begin{aligned}
\left|\nabla \beta_{i} \cdot \hat{x}\right| & \geq\left|\nabla \beta_{i} \cdot x_{1}\right|-\left|\nabla \beta_{i} \cdot x_{2}\right| \geq\left(\nabla \beta_{i} \cdot\left(\widehat{q-q_{i}}\right)\right)\left\|x_{1}\right\|-\left\|\nabla \beta_{i}\right\|\left\|x_{2}\right\| \\
& \geq\left\|x_{1}\right\|\left(\nabla \beta_{i} \cdot\left(\widehat{q-q_{i}}\right)-\frac{1}{\sqrt{2}} \nabla \beta_{i} \cdot\left(\widehat{q-q_{i}}\right)\right)>\frac{1}{5} \nabla \beta_{i} \cdot\left(\widehat{q-q_{i}}\right),
\end{aligned}
$$

since $1-\frac{1}{\sqrt{2}}>\frac{1}{4}$ and $\left\|x_{1}\right\| \geq \sqrt{\frac{2}{3}}$.

Recall that $\mathscr{S}_{i}(\varepsilon)$ was defined as the set

$$
\mathscr{S}_{i}(\varepsilon)=\left\{q \in \mathscr{F}: 0 \leq \beta_{i}(q) \leq \varepsilon\right\},
$$

where $\varepsilon$ is a positive constant such that $\varepsilon \leq E_{i}$. This set is a neighborhood in $\mathscr{F}$ about $\partial \mathscr{O}_{i}$. 
In the next lemma we will make use of the following technical definition. Let $H_{i}$ be the matrix valued function defined on $\mathscr{F}$ by

$$
\begin{aligned}
& H_{i}(q, \lambda) \triangleq\left(1-\sigma_{i}\right) I \\
& \quad+\sum_{j=0, j \neq i}^{M}\{\sigma_{j}\left(\nu_{j}-1\right) I+\sigma_{j}\left(q-q_{j}\right) \nabla \nu_{j}^{T}+\underbrace{\frac{\lambda \beta_{i}}{\left(\gamma_{d} \bar{\beta}_{j}+\lambda \beta_{j}\right)^{2}}}_{(*)} X_{i j}(q)\},
\end{aligned}
$$

where $X_{i j}$ for $i, j \in\{0, \ldots, M\}$, are arbitrary continuous matrix valued functions defined on $\mathscr{F}$. Recall that at each $q \in \mathscr{F}, S_{q}^{n}$ denotes the unit $n$-sphere in $T_{q} \mathscr{F}$. Consider the subset of $T_{q} \mathscr{F} \times T_{q} \mathscr{F}$ defined by

$$
\mathscr{D}_{q} \triangleq\left\{(\hat{u}, \hat{v}) \in S_{q}^{n} \times S_{q}^{n}: \hat{u} \cdot \hat{v}>c\right\},
$$

for some constant $0<c<1$. This set is a neighborhood of the diagonal in $S_{q}^{n} \times S_{q}^{n}$, that is,

$$
\mathscr{D}_{q} \supset\left\{(\hat{u}, \hat{u}): \hat{u} \in S_{q}^{n}\right\} .
$$

The motivation for the following lemma becomes clear if one identifies the term $(*)$ in $(31)$ as

$$
\frac{\lambda \beta_{i}}{\left(\gamma_{d} \bar{\beta}_{j}+\lambda \beta_{j}\right)^{2}} X_{i j}(q)=\nabla \sigma_{j}-\frac{\lambda \gamma_{d} \bar{\beta}_{i}}{\left(\gamma_{d} \bar{\beta}_{j}+\lambda \beta_{j}\right)^{2}} \nabla \beta_{i},
$$

i.e., it is the component of $\nabla \sigma_{j}$ which vanishes on $\partial \mathscr{O}_{i}$. Realizing that all the terms in (31) share $\beta_{i}$ as a common factor, we factor $\beta_{i}$ out and observe that

$$
\left(1-\sigma_{i}\right) \rightarrow \frac{\lambda}{\gamma_{d} \bar{\beta}_{i}}, \quad \sigma_{j} \rightarrow \frac{\gamma_{d} \bar{\beta}_{j}}{\lambda \beta_{j}}, \quad(*) \rightarrow \frac{1}{\lambda \beta_{j}^{2}} \quad \text { as } q \rightarrow \partial \mathscr{O}_{i} .
$$

Since in the set $\mathscr{S}_{i}(\varepsilon)$ we have that $\beta_{j}>E_{j}$ for $j \neq i$, and $\gamma_{d}>E_{d}$, it seems plausible that by making $\lambda$ sufficiently high, the matrix $\left(1-\sigma_{i}\right) I$ will dominate in (31), despite the fact that all the terms in (31) vanish as $q \rightarrow \partial \mathscr{O}_{i}$, since $\partial \mathscr{O}_{i}=\beta_{i}^{-1}(0)$. Note however that

$$
\nabla \sigma_{j} \rightarrow-\frac{1}{\lambda \beta_{j}} \nabla \beta_{i} \quad \text { as } q \rightarrow \partial \mathscr{O}_{i},
$$

since $\partial \mathscr{O}_{i}$ is a level set of $\sigma_{j}$. We consider the contribution of this component of $\nabla \sigma_{j}$ for $j \neq i$ in Lemma B.8 below.

Let $\mathbf{R}_{+}$denote the positive real numbers.

Lemma B.7. There exists a continuous function $\Lambda_{i}^{*}: \mathscr{S}_{i}\left(E_{i}\right) \rightarrow \mathbf{R}_{+}$, such that if $\lambda \geq \Lambda^{*}(q)$, then the bilinear form associated with $H_{i}(q, \lambda)$ is positive semidefinite on $\mathscr{D}_{q}$. That is, for all $(\hat{u}, \hat{v}) \in \mathscr{D}_{q}$,

$$
\hat{u}^{T}\left[H_{i}(q, \lambda)\right] \hat{v} \geq 0 .
$$


Moreover, equality is attained only when $q \in \partial \mathscr{O}_{i}$.

Proof. Using the definition of $\sigma_{i}(6)$,

$$
1-\sigma_{i}=\frac{\lambda \beta_{i}}{\gamma_{d} \bar{\beta}_{i}+\lambda \beta_{i}}
$$

Substituting for $\left(1-\sigma_{i}\right)$ and expanding $\sigma_{j}, j \neq i$, in (31) yields

$$
\begin{aligned}
H_{i}(q, \lambda) & \beta_{i} \cdot \underbrace{\left(\frac{1}{2} \frac{\lambda}{\gamma_{d} \bar{\beta}_{i}+\lambda \beta_{i}} I+\sum_{j=0, j \neq i}^{M} \frac{\gamma_{d} \bar{\beta}_{i, j}}{\gamma_{d} \bar{\beta}_{j}+\lambda \beta_{j}}\left\{\left(\nu_{j}-1\right) I+\left(q-q_{j}\right) \nabla \nu_{j}^{T}\right\}\right)}_{H_{i 2}(q, \lambda)} \\
& +\lambda \beta_{i} \cdot \underbrace{M}_{H_{i} \cdot \underbrace{\left(\frac{1}{2} \frac{1}{\gamma_{d} \bar{\beta}_{i}+\lambda \beta_{i}} I+\lambda \bar{\beta}_{j}\right)^{2}}_{j=0, j \neq i} X_{i j}(q))},
\end{aligned}
$$

where $\bar{\beta}_{i, j}$ denotes the "omitted product" $\prod_{k=0, k \neq i, j}^{M} \beta_{k}$. We will show that

$$
\hat{u}^{T}\left[H_{i 1}(q, \lambda)\right] \hat{v}>0 \quad \text { and } \quad \hat{u}^{T}\left[H_{i 2}(q, \lambda)\right] \hat{v}>0 \quad \text { for all }(\hat{u}, \hat{v}) \in \mathscr{D}_{q} .
$$

First consider $H_{i 1}$,

$$
\begin{aligned}
& \hat{u}^{T}\left[H_{i 1}(q, \lambda)\right] \hat{v} \\
& \quad>\frac{1}{2} \frac{c \lambda}{\gamma_{d} \bar{\beta}_{i}}+\lambda \beta_{i}+\sum_{j=0, j \neq i}^{M} \frac{\gamma_{d} \bar{\beta}_{i, j}}{\gamma_{d} \bar{\beta}_{j}+\lambda \beta_{j}} \underbrace{\hat{u}^{T}\left\{\left(\nu_{j}-1\right) I+\left(q-q_{j}\right) \nabla \nu_{j}^{T}\right\} \hat{v}}_{\tau_{j}(q)},
\end{aligned}
$$

since $(\hat{u}, \hat{v}) \in \mathscr{D}_{q}$. A sufficient condition for the positive definiteness of $H_{1 i}$ is

$$
\frac{1}{2} \frac{c \lambda}{\gamma_{d} \bar{\beta}_{i}+\lambda \beta_{i}}-\sum_{j=0, j \neq i}^{M} \frac{\gamma_{d} \bar{\beta}_{i, j}}{\gamma_{d} \bar{\beta}_{j}+\lambda \beta_{j}}, \quad\left|\tau_{j}\right| \geq 0
$$

which is implied by the inequality

$$
\frac{1}{2} \frac{c \lambda}{\gamma_{d} \bar{\beta}_{i}+\lambda \beta_{i}}-\sum_{j=0, j \neq i}^{M} \frac{\gamma_{d} \bar{\beta}_{i, j}}{\lambda \beta_{j}}, \quad\left|\tau_{j}\right| \geq 0
$$

or, equivalently, after multiplication by $2 \lambda\left(\gamma_{d} \bar{\beta}_{i}+\lambda \beta_{i}\right)$,

$$
c \lambda^{2}-\left(2 \beta_{i} \sum_{j=0, j \neq i}^{M} \frac{\gamma_{d} \bar{\beta}_{i, j}}{\beta_{j}}\left|\tau_{j}\right|\right) \lambda-2 \sum_{j=0, j \neq i}^{M}\left(\gamma_{d} \bar{\beta}_{i, j}\right)^{2}, \quad\left|\tau_{j}\right| \geq 0 .
$$


Solving for $\lambda$ and using the fact that in $\mathscr{S}_{i}\left(E_{i}\right), \beta_{i} \leq E_{i}$ and $\beta_{j} \geq E_{j}$ for $j \neq i$, yields the sufficient condition

$$
\begin{aligned}
\lambda \geq \frac{1}{c}\left\{E_{i} \sum_{j=0, j \neq i}^{M} \frac{\gamma_{d} \bar{\beta}_{i, j}}{E_{j}}\left|\tau_{j}\right|\right. & \\
& \left.+\sqrt{\left(E_{i} \sum_{j=0, j \neq i}^{M} \frac{\gamma_{d} \bar{\beta}_{i, j}}{E_{j}}\left|\tau_{j}\right|\right)^{2}+2 \sum_{j=0, j \neq i}^{M}\left(\gamma_{d} \bar{\beta}_{i, j}\right)^{2}\left|\tau_{j}\right|}\right\} \triangleq \Lambda_{i 1}^{*}(q),
\end{aligned}
$$

where we have used the fact that $c<1$. We turn now to $H_{i 2}$. Since

$$
\frac{1}{\left(\gamma_{d} \bar{\beta}_{j}+\lambda \beta_{j}\right)^{2}} \leq \frac{1}{\left(\lambda \beta_{j}\right)^{2}}
$$

and $(\hat{u}, \hat{v}) \in \mathscr{D}_{q}$, we have that

$$
\hat{u}^{T}\left[H_{i 2}(q, \lambda)\right] \hat{v}>\frac{1}{2} \frac{c}{\gamma_{d} \bar{\beta}_{i}+\lambda \beta_{i}}-\frac{1}{\lambda^{2}} \sum_{j=0, j \neq i}^{M} \frac{\left|\hat{u}^{T} X_{i j} \hat{v}\right|}{\beta_{j}^{2}} .
$$

Thus, similarly to the previous multiplication, the positive definiteness of $H_{i 2}$ is implied by the inequality

$$
c \lambda^{2}-\left(2 \beta_{i} \sum_{j=0, j \neq i}^{M} \frac{\left|\hat{u}^{T} X_{i j} \hat{v}\right|}{\beta_{j}^{2}}\right) \lambda-2 \gamma_{d} \bar{\beta}_{i} \sum_{j=0, j \neq i}^{M} \frac{\left|\hat{u}^{T} X_{i j} \hat{v}\right|}{\beta_{j}^{2}} \geq 0 .
$$

Solving for $\lambda$ and using the fact that in $\mathscr{S}_{i}\left(E_{i}\right), \beta_{i} \leq E_{i}$ and $\beta_{j} \geq E_{j}, j \neq i$, yields the sufficient condition

$$
\begin{aligned}
\lambda \geq \frac{1}{c}\left\{E_{i} \sum_{j=0, j \neq i}^{M} \frac{\left|\hat{u}^{T} X_{i j} \hat{v}\right|}{E_{j}^{2}}\right. & \\
& +\sqrt{\left.\left(E_{i} \sum_{j=0, j \neq i}^{M} \frac{\left|\hat{u}^{T} X_{i j} \hat{v}\right|}{E_{j}^{2}}\right)^{2}+2 \gamma_{d} \bar{\beta}_{i} \sum_{j=0, j \neq i}^{M} \frac{\mid \hat{u}^{T} X_{i j} \hat{v}}{E_{j}^{2}}\right\} \triangleq \Lambda_{i 2}^{*}(q) .}
\end{aligned}
$$

Finally, letting

completes the proof.

$$
\Lambda_{i}^{*}(q) \triangleq \max \left\{\Lambda_{i 1}^{*}(q), \Lambda_{i 2}^{*}(q)\right\}
$$

The following lemmas are used in Proposition 3.6.

Let $\left(q-q_{i}\right)$ be a vector based at $q \in E^{n}$. Consider the tangent space to $\mathscr{F}$ at $q, T_{q} \mathscr{F}$, as the orthogonal direct sum

$$
T_{q} \mathscr{F}=\left\langle q-q_{i}\right\rangle \oplus\left\langle q-q_{i}\right\rangle^{\perp} .
$$


Each vector $x \in T_{q} \mathscr{F}$ can be uniquely written as

$$
x=x_{1}+x_{2} \text { such that } x_{1} \in\left\langle q-q_{i}\right\rangle \text { and } x_{2} \in\left\langle q-q_{i}\right\rangle^{\perp} .
$$

Recall that the "cone" $\mathscr{C}_{q}$ was defined in equation (20) as the subset of $T_{q} \mathscr{F}$,

$$
\mathscr{C}_{q}=\left\{x \in S_{q}^{n}: \frac{\left\|x_{1}\right\|}{\left\|x_{2}\right\|}>\frac{\sqrt{2}}{{\widehat{\nabla \beta_{i}} \cdot\left(\widetilde{q-q_{i}}\right)}}\right\} .
$$

First we sketch the motivation for this particular decompositoin of $S_{q}^{n}$ into $\mathscr{C}_{q}$ and its complement. Since $h_{\lambda}$ depends linearly on the switches $\sigma_{j}$, its Jacobian depends linearly on $\sigma_{j}$ and $\nabla \sigma_{j}$ for $j \in\{0, \ldots, M\}$. Furthermore, each switch $\sigma_{j}$ is essentially a quotient of products of the various obstacle functions $\beta_{j}$ for $j \in\{0, \ldots, M\}$. Thus each $\nabla \sigma_{j}$ can be written as

$$
\nabla \sigma_{j}=s_{j i} \nabla \beta_{i}+\beta_{i} v_{j i}
$$

where $s_{j i}$ and $v_{j i}$ are, respectively, scalar and vector valued functions which depend on all the other obstacle functions. Expanding $\nabla \sigma_{j}$ for $j \in\{0, \ldots, M\}$ and using equation (28) for $\nabla \nu_{i}$, the Jacobian of $h_{\lambda}$ given in Lemma B.2 can be written in the form

$$
\begin{aligned}
D h_{\lambda}(q)= & \sigma_{i} \nu_{i} I-\sigma_{i} \nu_{i}\left(q-q_{i}\right)\left(q-q_{i}\right)^{T}+\alpha_{i}\left(q-q_{i}\right) \nabla \beta_{i}^{T} \\
& +\sum_{j=0, j \neq i}^{M} s_{j i}\left(q-q_{j}\right) \nabla \beta_{i}^{T}+\beta_{i} F_{i}(q, \lambda),
\end{aligned}
$$

where $\alpha_{i}$ and $F_{i}$ are, respectively, scalar and matrix valued functions which depend on all the other obstacle functions. In order to prove the nonsingularity of $D h_{\lambda}$ in the set near the $i$ th obstacle, $\mathscr{S}_{i}(\varepsilon)=\beta_{i}^{-1}[0, \varepsilon] \cap \mathscr{F}$, the fact that

$$
\operatorname{ker}\left[I-\left(\widehat{q-q_{i}}\right)\left(\widehat{q-q_{i}}\right)^{T}\right]=\left\langle q-q_{i}\right\rangle,
$$

necessitates a special consideration of this subspace. In fact, we have proved in Lemma B.6 that $\mathscr{C}_{q}-$ a "cone" neighborhood about $\left\langle q-q_{i}\right\rangle$, is contained in the positive cone of $\left[\left(q-q_{i}\right) \nabla \beta_{i}^{T}\right]$. Thus it seems plausible that by shrinking the set $\mathscr{S}_{i}(\varepsilon)$ toward $\partial \mathscr{O}_{i}$ i.e., by decreasing $\varepsilon$ and making $\left\|\nabla \sigma_{j}\right\|, j \neq i$, sufficiently small by increasing $\lambda$, the term $\alpha_{i}\left[\left(q-q_{i}\right) \nabla \beta_{i}^{T}\right]$ can be made dominant in (34). This is made precise in the following lemma.

Lemma B.8. Let $\varepsilon_{i 0}$ be a positive constant such that

$$
\nabla \beta_{i} \cdot\left(q-q_{i}\right)>0 \text { for all } q \in \mathscr{S}_{i}\left(\varepsilon_{i 0}\right) .
$$

Given a valid arrangement of $\mathscr{F}$, and a suitable $\mathscr{M}$, there exist two constants $\varepsilon_{i 1} \leq \varepsilon_{i 0}$ and $\Lambda_{i 1}$, such that for all $\varepsilon \leq \varepsilon_{i 1}$ and $q \in \mathscr{S}_{i}(\varepsilon)$, if $\lambda \geq \Lambda_{i 1}$, then

$$
\hat{x}^{T}\left[D h_{\lambda}(q)\right] \hat{x}>0 \text { for all } \hat{x} \in \mathscr{C}_{q} \text {. }
$$


Proof. Distinguishing the $i$ th obstacle terms in the formula for the Jacobian of $h_{\lambda}$ given in Lemma B.2 yields

$$
\begin{aligned}
& D h_{\lambda}(q)=\underbrace{\left(\nu_{i}-1\right)\left(q-q_{i}\right) \nabla \sigma_{i}^{T}}_{D h_{1}(q, \lambda)} \\
& +\underbrace{\left(1-\sigma_{i}+\sigma_{i} \nu_{i}\right) I+\sigma_{i}\left(q-q_{i}\right) \nabla \nu_{i}^{T}+\sum_{j=0, j \neq i}^{M}\left\{\sigma_{j}\left(\nu_{j}-1\right) I+\left(q-q_{j}\right)\left(\sigma_{j} \nabla \nu_{j}+\left(\nu_{j}-1\right) \nabla \sigma_{j}\right)^{T}\right\}}_{D h_{2}(q, \lambda)}
\end{aligned}
$$

We will show that

$$
\hat{x}^{T}\left[D h_{1}(q, \lambda)\right] \hat{x} \geq 0 \text { and } \hat{x}^{T}\left[D h_{2}(q, \lambda)\right] \hat{x}>0 \text { for all } \hat{x} \in \mathscr{C}_{q},
$$

this would imply that $\hat{x}^{T}\left[D h_{\lambda}(q)\right] \hat{x}>0$. We begin with $D h_{1}$. Using equation (29) to expand $\nabla \sigma_{i}$,

$$
\begin{aligned}
\hat{x}^{T}\left[D h_{1}(q)\right] \hat{x} & =\lambda \frac{1-\nu_{i}}{\left(\gamma_{d} \bar{\beta}_{i}+\lambda \beta_{i}\right)^{2}}\{\gamma_{d} \bar{\beta}_{i} \overbrace{\hat{x}}^{T}\left[\left(q-q_{i}\right) \nabla \beta_{i}^{T}\right] \hat{x}-\beta_{i} \hat{x}^{T}\left[\left(q-q_{i}\right) \nabla\left(\gamma_{d} \bar{\beta}_{i}\right)^{T}\right] \hat{x}\} \\
& =\lambda \frac{1-\nu_{i}}{\left(\gamma_{d} \bar{\beta}_{i}+\lambda \beta_{i}\right)^{2}}\left(\hat{x} \cdot\left(q-q_{i}\right)\right)\left\{\gamma_{d} \bar{\beta}_{i}\left(\nabla \beta_{i} \cdot \hat{x}\right)-\beta_{i}\left(\nabla\left(\gamma_{d} \bar{\beta}_{i}\right) \cdot \hat{x}\right)\right\} .
\end{aligned}
$$

According to Lemma B.6, the term $(*)$ is nonnegative in the set $\mathscr{S}_{i}\left(\varepsilon_{i 0}\right)$. Furthermore, according to Lemma 3.5 we can assume that $\mathscr{M}$ satisfies the containment condition in $\mathscr{S}_{i}\left(E_{i}\right)$,

$$
\nu_{i}(q) \leq 1 \text { for all } q \in \mathscr{S}_{i}\left(E_{i}\right) .
$$

Therefore, it follows from (36) that a sufficient condition for the positive semidefiniteness of $D h_{1}$ is

$$
\left|\nabla \beta_{i} \cdot \hat{x}\right| \geq \frac{\beta_{i}}{\gamma_{d} \bar{\beta}_{i}}\left\|\nabla\left(\gamma_{d} \bar{\beta}_{i}\right)\right\|,
$$

which is implied in turn by

$$
\left|\nabla \beta_{i} \cdot \hat{x}\right| \geq \beta_{i}\left(\sum_{j=0, j \neq i} \frac{\left\|\nabla \beta_{j}\right\|}{\beta_{j}}+\frac{\left\|\nabla \gamma_{d}\right\|}{\gamma_{d}}\right),
$$

since $\left\|\nabla\left(\gamma_{d} \bar{\beta}_{i}\right)\right\| \leq \sum_{j=0, j \neq i}^{M} \gamma_{d} \bar{\beta}_{i, j}\left\|\nabla \beta_{j}\right\|+\bar{\beta}_{i}\left\|\nabla \gamma_{d}\right\|$. Note that $\hat{x} \in \mathscr{C}_{q}$ implies that $\nabla \beta_{i} \cdot \hat{x} \neq 0$, thus there is no problem with (37). Using the lower bound on $\left|\nabla \beta_{i} \cdot \hat{x}\right|$ given in Lemma B.6, and the identity $\left\|\nabla \gamma_{d}\right\|=2 \sqrt{\gamma_{d}}$, which follows from equation (5), we obtain the stricter inequality,

$$
\frac{1}{5}\left(\widehat{q-q_{i}}\right) \cdot \nabla \beta_{i} \geq \beta_{i}\left(\sum_{j=0, j \neq i} \frac{\left\|\nabla \beta_{j}\right\|}{\beta_{j}}+\frac{2}{\sqrt{\gamma_{d}}}\right),
$$


or,

$$
\beta_{i} \leq \frac{1}{5} \frac{\left(q-q_{i}\right) \cdot \nabla \beta_{i}}{\left\|q-q_{i}\right\|\left(\sum_{j=0, j \neq i} \frac{\left\|\nabla \beta_{j}\right\|}{\beta_{j}}+\frac{2}{\sqrt{\gamma_{d}}}\right)} .
$$

Thus, the condition,

(38) $\varepsilon \leq \min \left\{\frac{1}{5} \frac{\min _{\mathscr{S}_{(}\left(\varepsilon_{i 0}\right)}\left\{\left(q-q_{i}\right) \cdot \nabla \beta_{i}\right\}}{\max _{\mathscr{F}}\left\{\left\|q-q_{i}\right\|\right\}\left(\sum_{j=0, j \neq i} \frac{\max _{\mathscr{F}}\left\{\left\|\nabla \beta_{j}\right\|\right\}}{E_{j}}+\frac{2}{E_{d}}\right)}, \varepsilon_{i 0}\right\} \triangleq \varepsilon_{i 1}^{\prime}$,

guarantees the positive semidefiniteness of $D h_{1}$ at each $q \in \mathscr{S}_{i}(\varepsilon)$ and $\hat{x} \in \mathscr{C}_{q}$. We turn now to $D h_{2}$. Using Lemma B.3 to expand $\nabla \nu_{i}$ yields

$$
\begin{aligned}
D h_{2}(q, \lambda) & =\underbrace{\sigma_{i} \nu_{i} I-\sigma_{i} \nu_{i}\left(q \widehat{-q_{i}}\right)\left(\widehat{q-q_{i}}\right)^{T}}_{D h_{21}(q, \lambda)} \\
& +\underbrace{\frac{\sigma_{i} \nu_{i}}{1+\beta_{i}}\left(q-q_{i}\right) \nabla \beta_{i}^{T}+\left(1-\sigma_{i}\right) I+\sum_{j=0, j \neq i}^{M}\left\{\sigma_{j}\left(\nu_{j}-1\right) I+\left(q-q_{j}\right)\left(\sigma_{j} \nabla \nu_{j}+\left(\nu_{j}-1\right) \nabla \sigma_{j}\right)^{T}\right\}}_{D h_{22}(q, \lambda)} .
\end{aligned}
$$

We will show that

$$
\hat{x}^{T}\left[D h_{21}(q, \lambda)\right] \hat{x} \geq 0 \text { and } \hat{x}^{T}\left[D h_{22}(q, \lambda)\right] \hat{x}>0 \text { for all } \hat{x} \in \mathscr{C}_{q},
$$

this would imply the positive definiteness of $D h_{2}$. First,

$$
\begin{aligned}
\hat{x}^{T}\left[D h_{21}(q, \lambda)\right] \hat{x} & =\sigma_{i} \nu_{i} \hat{x}^{T}\left[I-\left(\widehat{q-q_{i}}\right)\left(\widehat{q-q_{i}}\right)^{T}\right] \hat{x} \\
& =\sigma_{i} \nu_{i}\left(1-\left(\left(q \widehat{-q_{i}}\right) \cdot \hat{x}\right)^{2}\right) \geq 0 .
\end{aligned}
$$

Second, using equation (29), repeated here,

$$
\nabla \sigma_{j}=\frac{\lambda}{\left(\gamma_{d} \bar{\beta}_{j}+\lambda \beta_{j}\right)^{2}}\left(\beta_{j} \nabla\left(\gamma_{d} \bar{\beta}_{j}\right)-\gamma_{d} \bar{\beta}_{j} \nabla \beta_{j}\right)
$$

expand $\nabla\left(\gamma_{d} \bar{\beta}_{j}\right)=\gamma_{d} \bar{\beta}_{i, j} \nabla \beta_{i}+\beta_{i} \nabla\left(\gamma_{d} \bar{\beta}_{i, j}\right)$, to emphasize the dependence on $\nabla \beta_{i}$

$$
\begin{aligned}
\left(\nu_{j}-1\right) \nabla \sigma_{j}= & \left(\nu_{j}-1\right) \frac{\lambda \gamma_{d} \bar{\beta}_{i}}{\left(\gamma_{d} \bar{\beta}_{j}+\lambda \beta_{j}\right)^{2}} \nabla \beta_{i} \\
& +\frac{\lambda \beta_{i}}{\left(\gamma_{d} \bar{\beta}_{j}+\lambda \beta_{j}\right)^{2}} \underbrace{\left(\nu_{j}-1\right)\left\{\beta_{j} \nabla\left(\gamma_{d} \bar{\beta}_{i, j}\right)-\gamma_{d} \bar{\beta}_{i, j} \nabla \beta_{j}\right\}}_{X_{i j}(q)} .
\end{aligned}
$$


Substituting for $\nabla \sigma_{j}$ in $D h_{22}$ yields

$$
\begin{aligned}
D h_{22}(q, \lambda)= & \underbrace{\frac{\sigma_{i} \nu_{i}}{1+\beta_{i}}\left(q-q_{i}\right) \nabla \beta_{i}^{T}+\left(\sum_{j=0, j \neq i}^{M}\left(\nu_{j}-1\right) \frac{\lambda \gamma_{d} \bar{\beta}_{i}}{\left(\gamma_{d} \bar{\beta}_{j}+\lambda \beta_{j}\right)^{2}}\left(q-q_{j}\right)\right) \nabla \beta_{i}^{T}}_{D h_{221}(q, \lambda)} \\
& +\underbrace{\left(1-\sigma_{i}\right) I+\sum_{j}^{M}\left\{\sigma_{j}\left(\nu_{j}-1\right) I+\sigma_{j}\left(q-q_{j}\right) \nabla \nu_{j}^{T}+\frac{\lambda \beta_{i}}{\left(\gamma_{d} \bar{\beta}_{j}+\lambda \beta_{j}\right)^{2}} X_{i j}(q)\right\}}_{j=0, j \neq i} .
\end{aligned}
$$

We will show that

$$
\hat{x}^{T}\left[D h_{221}(q)\right] \hat{x}>0 \text { and } \hat{x}^{T}\left[D h_{222}(q, \lambda)\right] \hat{x} \geq 0 \text { for all } q \in \mathscr{C}_{q} .
$$

First,

$$
\begin{aligned}
& \hat{x}^{T}\left[D h_{221}(q, \lambda)\right] \hat{x}=\frac{\sigma_{i} \nu_{i}}{1+\beta_{i}} \overbrace{\hat{x}^{T}\left[\left(q-q_{i}\right) \nabla \beta_{i}^{T}\right] \hat{x}}^{(*)} \\
& \quad+\lambda \gamma_{d} \bar{\beta}_{i} \sum_{j=0, j \neq i}^{M} \frac{\left(\nu_{j}-1\right)}{\left(\gamma_{d} \bar{\beta}_{j}+\lambda \beta_{j}\right)^{2}} \hat{x}^{T}\left[\left(q-q_{j}\right) \nabla \beta_{i}^{T}\right] \hat{x} .
\end{aligned}
$$

According to Lemma B.6, the term $(*)$ is positive in $\mathscr{S}_{i}\left(\varepsilon_{i 0}\right)$, therefore the positive definiteness of $D h_{221}$ is implied by the inequality,

$$
\frac{\sigma_{i} \nu_{i}}{1+\beta_{i}}\left|\hat{x} \cdot\left(q-q_{i}\right)\right|+\lambda \gamma_{d} \bar{\beta}_{i} \sum_{j=0, j \neq i}^{M} \frac{\left(\nu_{j}-1\right)}{\left(\gamma_{d} \bar{\beta}_{j}+\lambda \beta_{j}\right)^{2}} \hat{x} \cdot\left(q-q_{j}\right)>0,
$$

obtained by factoring out the term $\left(\nabla \beta_{i} \cdot \hat{x}\right)$. Writing $x=x_{1}+x_{2}$, where $x_{1} \in\left\langle q-q_{i}\right\rangle$ and $x_{2} \in\left\langle q-q_{i}\right\rangle^{\perp}$, the last inequality is implied in turn by

$$
\sigma_{i} \nu_{i}\left\|x_{1}\right\|\left\|q-q_{i}\right\|>\lambda \gamma_{d} \bar{\beta}_{i}\left(1+\beta_{i}\right) \sum_{j=0, j \neq i}^{M} \frac{\left|\nu_{j}-1\right|}{\left(\gamma_{d} \bar{\beta}_{j}+\lambda \beta_{j}\right)^{2}}\left\|q-q_{j}\right\|,
$$

where we have used the identity $\left|\hat{x} \cdot\left(q-q_{i}\right)\right|=\left|\left(x_{1}+x_{2}\right) \cdot\left(q-q_{i}\right)\right|=\left\|x_{1}\right\|\left\|q-q_{i}\right\|$. According to Lemma B.6, at any point $q \in \mathscr{S}_{i}\left(\varepsilon_{i 0}\right)$ and for any $\hat{x} \in \mathscr{C}_{q}$, we have that $\left\|x_{1}\right\| \geq\left\|x_{1}\right\|^{2} \geq \frac{2}{3}$; thus we obtain the stricter inequality

$$
\frac{2}{3} \frac{\nu_{i}}{\gamma_{d} \bar{\beta}_{i}+\lambda \beta_{i}}\left\|q-q_{i}\right\| \geq \lambda\left(1+\beta_{i}\right) \sum_{j=0, j \neq i}^{M} \frac{\left|\nu_{j}-1\right|}{\left(\gamma_{d} \bar{\beta}_{j}+\lambda \beta_{j}\right)^{2}}\left\|q-q_{j}\right\|,
$$

where we have expanded $\sigma_{i}$. Since,

$$
\frac{1}{\left(\gamma_{d} \bar{\beta}_{j}+\lambda \beta_{j}\right)^{2}} \leq \frac{1}{\left(\lambda \beta_{j}\right)^{2}},
$$

the inequality (40) is implied by the stricter inequality,

$$
\frac{2}{3} \nu_{i}\left\|q-q_{i}\right\| \lambda \geq\left(1+\beta_{i}\right)\left(\gamma_{d} \bar{\beta}_{i}+\lambda \beta_{i}\right) \sum_{j=0, j \neq i}^{M} \frac{\left|\nu_{j}-1\right|}{\beta_{j}^{2}}\left\|q-q_{j}\right\|,
$$


which is implied in turn by the two inequalities,

$$
\frac{1}{3} \nu_{i}\left\|q-q_{i}\right\| \lambda \geq\left(1+\beta_{i}\right) \gamma_{d} \bar{\beta}_{i} \sum_{j=0, j \neq i}^{M} \frac{\left|\nu_{j}-1\right|}{\beta_{j}^{2}}\left\|q-q_{j}\right\|,
$$

and,

$$
\frac{1}{3} \nu_{i}\left\|q-q_{i}\right\| \geq\left(1+\beta_{i}\right) \beta_{i} \sum_{j=0, j \neq i}^{M} \frac{\left|\nu_{j}-1\right|}{\beta_{j}^{2}}\left\|q-q_{j}\right\| .
$$

It follows from the definition of $\mathscr{S}_{i}(\varepsilon)$ that for all $\varepsilon \leq E_{i}$ we have that $\mathscr{S}_{i}(\varepsilon) \subset$ $\mathscr{S}_{i}\left(E_{i}\right)$; this, together with the fact that in $\mathscr{S}_{i}\left(E_{i}\right), \beta_{i} \leq E_{i}$ and $\beta_{j} \geq E_{j}$, yields the sufficient condition,

$$
\lambda \geq 3\left(1+E_{i}\right) \frac{\max _{\mathscr{F}}\left\{\gamma_{d} \bar{\beta}_{i} \sum_{j=0, j \neq i}^{M} \frac{\left|\rho_{j}\left(\beta_{j}+1\right)-1\right|}{\left(E_{j}\right)^{2}}\right\}}{\min _{\mathscr{S}_{i}\left(E_{i}\right)}\left\{\nu_{i}\left\|q-q_{i}\right\|\right\}} \triangleq \Lambda_{i 1}^{\prime},
$$

and,

$$
\beta_{i} \leq \min \left\{\frac{1}{3\left(1+E_{i}\right)} \frac{\min _{\mathscr{S}_{i}\left(E_{i}\right)}\left\{\nu_{i}\left\|q-q_{i}\right\|\right\}}{\max _{\mathscr{F}}\left\{\sum_{j=0, j \neq i}^{M} \frac{\left|\rho_{j}\left(\beta_{j}+1\right)-1\right|}{\left(E_{j}\right)^{2}}\right\}}, \varepsilon_{i 1}^{\prime}\right\} \triangleq \varepsilon_{i 1},
$$

where we expanded $\left|\nu_{j}-1\right| \| q-q_{j}||=\left|\rho_{j}\left(\beta_{j}+1\right)-1\right|$. Note that by construction (Definition 7) $\nu_{i}$ is positive on $\mathscr{F}$, and

$$
\min _{\mathscr{S}_{i}\left(E_{i}\right)}\left\{\nu_{i}\left\|q-q_{i}\right\|\right\}=\min _{\mathscr{S}_{i}\left(E_{i}\right)}\left\{\rho_{i}\left(1+\beta_{i}(q)\right)\right\} \geq \rho_{i}
$$

We turn now to $D h_{222}$. Identifying $D h_{222}$ with $H_{i}(q, \lambda)$ from Lemma B.7, via

$$
\hat{u}=\hat{v}=\hat{x} \quad \text { and } \quad c=\hat{x} \cdot \hat{x}=1,
$$

and letting

$$
\Lambda_{i 1}^{\prime \prime} \triangleq \max _{\mathscr{S}_{i}\left(E_{i}\right)}\left\{\Lambda^{*}(q)\right\}
$$

where $\hat{u}, \hat{v}, c$ and $\Lambda^{*}$ are defined in this lemma; constitutes a guarantee that for all $q \in \mathscr{S}_{i}\left(E_{i}\right)$, if $\lambda \geq \Lambda_{i 1}^{\prime \prime}$, then

$$
\hat{x}^{T}\left[D h_{222}(q, \lambda)\right] \hat{x} \geq 0 \text { for all } \hat{x} \in \mathscr{C}_{q} .
$$

In particular, since $\mathscr{S}_{i}\left(\varepsilon_{i 1}\right) \subset \mathscr{S}_{i}\left(E_{i}\right)$, we conclude that if the parameter $\lambda$ is chosen by

$$
\lambda \geq \max \left\{\Lambda_{i 1}^{\prime}, \Lambda_{i 1}^{\prime \prime}\right\}
$$

then for all $\varepsilon \leq \varepsilon_{i 1}$ and $q \in \mathscr{S}_{i}(\varepsilon)$,

$$
\hat{x}^{T}\left[D h_{\lambda}(q)\right] \hat{x}>0 \quad \text { for all } \hat{x} \in \mathscr{C}_{q} \text {. }
$$


Consider the complementary case: at each tangent space, the test directions belong to the "cone" $S_{q}^{n}-\mathscr{C}_{q}$, in which

$$
\left\|x_{2}\right\| \geq \frac{1}{\sqrt{2}}\left[\left(q \widehat{-q}_{i}\right) \cdot \widehat{\nabla \beta}_{i}\right]\left\|x_{1}\right\| .
$$

The "cone" $S_{q}^{n}-\mathscr{C}_{q}$ is a neighborhood about the $(n-1)$-dimensional subspace $\left\langle q-q_{i}\right\rangle^{\perp}$. The intuitive motivation for the next lemma becomes clear from the following argument. Consider the "symmetric part" of $D h_{\lambda}$, defined as $\left(D h_{\lambda}\right)_{s} \triangleq \frac{1}{2}\left(D h_{\lambda}+D h_{\lambda}^{T}\right)$. Using (34), its restriction to the subspace $\left\langle q-q_{i}\right\rangle^{\perp}$ (translated to $T_{q} \mathscr{F}$ ) is of the form

$$
\left[D h_{\lambda}(q)\right]_{s} \mid\left\langle q-q_{i}\right\rangle^{\perp}=\nu_{i} I+\sum_{j=0, j \neq i}^{M} s_{j i}\left[\left(q-q_{j}\right) \nabla \beta_{i}^{T}\right]_{s}+\beta_{i} F_{i}(q, \lambda)_{s} .
$$

If $\left[D h_{\lambda}\right]_{s}>0$ on $\left\langle q-q_{i}\right\rangle^{\perp}$, the $\left[D h_{\lambda}\right] \hat{x} \neq 0$ for test directions $\hat{x} \in$ $\left\langle q-q_{i}\right\rangle^{\perp}$. Thus, by choosing $\varepsilon$ sufficiently small, the set $\mathscr{S}_{i}(\varepsilon)=\beta_{i}^{-1}[0, \varepsilon]$ can be shrinked about $\partial \mathscr{O}_{i}$ so that the matrix $\nu_{i} I$, which is positive definite by construction, becomes dominant in (45). This idea is extended to the cone $S_{q}^{n}-\mathscr{C}_{q}$ in the following lemma.

Lemma B.9. Let $\varepsilon_{i 0}$ be a positive constant such that

$$
\nabla \beta_{i} \cdot\left(q-q_{i}\right)>0 \text { for all } q \in \mathscr{S}_{i}\left(\varepsilon_{i 0}\right) \text {. }
$$

Given a valid arrangement of $\mathscr{F}$, and a suitable $\mathscr{M}$, there exist two constants, $\varepsilon_{i 2} \leq \varepsilon_{i 0}$ and $\Lambda_{i 2}$, such that for all $\varepsilon \leq \varepsilon_{i 2}$ and $q \in \mathscr{S}_{i}(\varepsilon)$, if $\lambda \geq \Lambda_{i 2}$, then

$$
x_{2}^{T}\left[D h_{\lambda}(q)\right] \hat{x}>0 \text { for all } \hat{x} \in S_{q}^{n}-\mathscr{C}_{q},
$$

where $\hat{x}=x_{1}+x_{2}$ such that $x_{1} \in\left\langle q-q_{i}\right\rangle$ and $x_{2} \in\left\langle q-q_{i}\right\rangle^{\perp}$.

Proof. First we establish a lower bound on $\left\|x_{2}\right\|$,

$$
1=\left\|x_{1}\right\|^{2}+\left\|x_{2}\right\|^{2} \leq\left(1+\frac{1}{\frac{1}{2}\left[\left(q \widehat{-q_{i}}\right) \cdot \widehat{\nabla \beta_{i}}\right]^{2}}\right)\left\|x_{2}\right\|^{2},
$$

which implies that

$$
\left\|x_{2}\right\|^{2} \geq \frac{\frac{1}{2}\left[\left(q-q_{i}\right) \cdot \widehat{\nabla \beta}_{i}\right]^{2}}{\frac{1}{2}\left[\left(q \widehat{-q_{i}}\right) \cdot{\widehat{\nabla \beta_{i}}}_{i}\right]^{2}+1} \geq \frac{1}{3}\left[\left(q \widehat{-q_{i}}\right) \cdot \widehat{\nabla \beta_{i}}\right]^{2} .
$$

Evaluating $D h_{\lambda}(q)$ (Lemma B.2) along $\hat{x}$, and grouping together all the terms in $\left[D h_{\lambda}(q)\right] \hat{x}$ which lie in $\left\langle q-q_{i}\right\rangle$, yields

$$
\begin{aligned}
{\left[D h_{\lambda}(q) \hat{x}=\right.} & \underbrace{\left(1-\sigma_{i}+\sigma_{i} \nu_{i}\right) x_{1}+\left\{\left(\nu_{i}-1\right)\left(\nabla \sigma_{i} \cdot \hat{x}\right)+\sigma_{i}\left(\nabla \nu_{i} \cdot \hat{x}\right)\right\}\left(q-q_{i}\right)}_{y_{1}} \\
& +\underbrace{\left(1-\sigma_{i}+\sigma_{i} \nu_{i}\right) x_{2}+\sum_{j=0, j \neq i}^{M}\left\{\sigma_{j}\left(\nu_{j}-1\right) I+\left(q-q_{j}\right)\left(\sigma_{j} \nabla \nu_{j}+\left(\nu_{j}-1\right) \nabla \sigma_{j}\right)^{T}\right\} \hat{x}}_{y_{2}} .
\end{aligned}
$$


Since $y_{1} \in\left\langle q-q_{i}\right\rangle$, we have that $y_{1} \cdot x_{2}=0$, and as a consequence,

$$
\begin{aligned}
\hat{x}_{2}^{T}[D h(q)] \hat{x}= & \hat{x}_{2} \cdot y_{2}=\overbrace{\left(1-\sigma_{i}+\sigma_{i} \nu_{i}\right)}^{(*)}\left\|x_{2}\right\| \\
& +\sum_{j=0, j \neq i}^{M} \hat{x}_{2}^{T}\left\{\sigma_{j}\left(\nu_{j}-1\right) I+\left(q-q_{j}\right)\left(\sigma_{j} \nabla \nu_{j}+\left(\nu_{j}-1\right) \nabla \sigma_{j}\right)^{T}\right\} \hat{x} .
\end{aligned}
$$

According to the containment condition,

$$
\nu_{i}(q) \leq 1 \text { for all } q \in \mathscr{S}_{i}\left(E_{i}\right),
$$

thus, the expression $(*)$ can be bounded from below by

$$
\sigma_{i} \nu_{i}+\left(1-\sigma_{i}\right)=\sigma_{i}\left(\nu_{i}-1\right)+1 \geq\left(\nu_{i}-1\right)+1=\nu_{i},
$$

since, by definition, $0 \leq \sigma_{i} \leq 1$. Using (39) to expand the $j$ th switch gradient, $\nabla \sigma_{j}$, yields

$$
\begin{gathered}
\hat{x}_{2}^{T}[D h(q)] \hat{x} \geq \underbrace{\frac{1}{2} \nu_{i}\left\|x_{2}\right\|+\lambda \gamma_{d} \bar{\beta}_{i}\left(\nabla \beta_{i} \cdot \hat{x}\right) \sum_{j=0, j \neq i}^{M} \frac{\nu_{j}-1}{\left(\gamma_{d} \bar{\beta}_{j}+\lambda \beta_{j}\right)^{2}} \hat{x}_{2} \cdot\left(q-q_{j}\right)}_{\kappa_{1}(q, \lambda)} \\
+\underbrace{\frac{1}{2} \nu_{i}\left\|x_{2}\right\|+\sum_{j=0, j \neq i}^{M} \hat{x}_{2}^{T}\left\{\sigma_{j}\left(\nu_{j}-1\right) I+\sigma_{j}\left(q-q_{j}\right) \nabla \nu_{j}^{T}+\frac{\lambda \beta_{i}}{\left(\gamma_{d} \bar{\beta}_{j}+\lambda \beta_{j}\right)^{2}} X_{i j}(q)\right\} \hat{x}}_{\kappa_{2}(q, \lambda)} .
\end{gathered}
$$

We will show that

$$
\kappa_{1}(q, \lambda)>0 \text { and } \kappa_{2}(q, \lambda) \geq 0 \text { for all } \hat{x} \in S_{q}^{n}-\mathscr{C}_{q} .
$$

First consider $\kappa_{1}$. Since

$$
\frac{1}{\left(\gamma_{d} \bar{\beta}_{j}+\lambda \beta_{j}\right)^{2}} \leq \frac{1}{\left(\lambda \beta_{j}\right)^{2}}
$$

the positive definiteness of $\kappa_{1}$ is implied by the inequality

$$
\frac{1}{2} \nu_{i}\left\|x_{2}\right\|-\frac{1}{\lambda} \gamma_{d} \bar{\beta}_{i}\left|\nabla \beta_{i} \cdot \hat{x}\right| \sum_{j=0, j \neq i}^{M} \frac{\left|\nu_{j}-1\right|}{\left(\beta_{j}\right)^{2}}\left\|q-q_{j}\right\|>0 .
$$

Since $\left|\nabla \beta_{i} \cdot \hat{x}\right| \leq\left|\nabla \beta_{i} \cdot x_{1}\right|+\left|\nabla \beta_{i} \cdot x_{2}\right|$ and $\left|\nabla \beta_{i} \cdot x_{1}\right|=\left\|x_{1}\right\| \nabla \beta_{i} \cdot\left(q \widehat{-q_{i}}\right)$, we obtain the stricter inequality

$$
\frac{1}{2} \nu_{i}\left\|x_{2}\right\|-\frac{1}{\lambda}\left\{\left\|x_{1}\right\| \nabla \beta_{i}\left(q-q_{i}\right)+\left\|x_{2}\right\|\left\|\nabla \beta_{i}\right\|\right\} \gamma_{d} \bar{\beta}_{i} \sum_{j=0, j \neq i}^{M} \frac{\left|\nu_{j}-1\right|}{\left(\beta_{j}\right)^{2}}\left\|q-q_{j}\right\|>0 .
$$

Using (44) to replace $\left\|x_{1}\right\|$ by its upper bound in $S_{q}^{n}-\mathscr{C}_{q}$, yields the stricter inequality

$$
\frac{\left\|x_{2}\right\|}{\lambda}\left\{\frac{1}{2} \lambda \nu_{i}-\left\|\nabla \beta_{i}\right\|(\sqrt{2}+1) \gamma_{d} \bar{\beta}_{i} \sum_{j=0, j \neq i}^{M} \frac{\left|\nu_{j}-1\right|}{\left(\beta_{j}\right)^{2}}\left\|q-q_{j}\right\|\right\}>0 .
$$


Thus we obtain the following sufficient condition for $\kappa_{1}>0$,

$$
\lambda>2(1+\sqrt{2}) \frac{\max _{\mathscr{F}}\left\{\gamma_{d} \bar{\beta}_{i}\left\|\nabla \beta_{j}\right\| \sum_{j=0, j \neq i}^{M} \frac{\left|\nu_{j}-1\right|}{\left(E_{j}\right)^{2}}\left\|q-q_{j}\right\|\right\}}{\min _{\mathscr{S}_{i}\left(E_{i 2}\right)}\left\{\nu_{i}\right\}} \triangleq \Lambda_{i 2}^{\prime},
$$

where we have used the fact that $\mathscr{S}_{i}\left(\varepsilon_{i 0}\right) \subset \mathscr{S}_{i}\left(E_{i}\right)$, since $\varepsilon_{i 0} \leq E_{i}$. Note that according to its definition, $\nu_{i}$ is positive on $\mathscr{F}$, in fact,

$$
\min _{\mathscr{S}_{i}\left(E_{i 2}\right)}\left\{\nu_{i}\right\}=\min _{\mathscr{S}_{i}\left(E_{i 2}\right)}\left\{\rho_{i} \frac{1+\beta_{i}(q)}{\left\|q-q_{i}\right\|}\right\} \geq \rho_{i} \frac{1}{\min _{\partial \mathscr{O}_{i}}\left\{\left\|q-q_{i}\right\|\right\}}>0,
$$

since $q_{i} \notin \partial \mathscr{O}_{i}$. We turn now to the term $\kappa_{2}$ in (48). Expanding $\sigma_{j}$ for $j \neq i$, and using $(33)$ yields

$$
\begin{aligned}
\frac{1}{2} \nu_{i}\left\|x_{2}\right\|+\beta_{i} \sum_{j=0, j \neq i}^{M} \hat{x}_{2}^{T}\left\{\frac { 1 } { \gamma _ { d } \overline { \beta } _ { j } + \lambda \beta _ { j } } \left(\left(\nu_{j}-1\right) I\right.\right. & \left.+\left(q-q_{j}\right) \nabla \nu_{j}^{T}\right) \\
& \left.+\frac{\lambda}{\left(\gamma_{d} \overline{\beta_{j}}+\lambda \beta_{j}\right)^{2}} X_{i j}(q)\right\} \hat{x}
\end{aligned}
$$

which implies that

$$
\begin{aligned}
\kappa_{2}(q, \lambda) \geq & \frac{1}{2} \nu_{i}\left\|x_{2}\right\| \\
& -\frac{\beta_{i}}{\lambda} \sum_{j=0, j \neq i}^{M}\left\{\frac{1}{\beta_{j}}\left|\nu_{j}-1\right|+\frac{1}{\beta_{j}}\left\|q-q_{j}\right\|\left\|\nabla \nu_{j}\right\|+\frac{1}{\left(\beta_{j}\right)^{2}}\left\|X_{i j}(q)\right\|\right\} .
\end{aligned}
$$

Using (46) to replace $\left\|x_{2}\right\|$ by its lower bound from equation (44) obtains the stricter inequality,

$$
\begin{aligned}
\frac{1}{2} \nu_{i} \frac{1}{\sqrt{3}}\left[\left(q-q_{i}\right)\right. & \left.\cdot \widehat{\nabla \beta}_{i}\right] \\
& \geq \frac{\beta_{i}}{\lambda} \sum_{j=0, j \neq i}^{M} \frac{1}{\beta_{j}}\left\{\left|\nu_{j}-1\right|+\left\|q-q_{j}\right\|\left\|\nabla \nu_{j}\right\|+\frac{1}{\beta_{j}}\left\|X_{i j}(q)\right\|\right\},
\end{aligned}
$$

which is implied in turn by the inequalities,

$$
\beta_{i} \leq \frac{1}{\sqrt{3}}\left[\left(q-q_{i}\right) \cdot \widehat{\nabla \beta}_{i}\right]
$$

and

$$
\lambda \geq 2 \sqrt{3} \frac{\sum_{j=0, j \neq i}^{M} \frac{1}{\beta_{j}}\left\{\frac{1}{\beta_{j}}\left|\nu_{j}-1\right|+\frac{1}{\beta_{j}}\left\|q-q_{j}\right\|\left\|\nabla \nu_{j}\right\|+\frac{1}{\left(\beta_{j}\right)^{2}}\left\|X_{i j}(q)\right\|\right\}}{\nu_{i}} .
$$

Thus, we obtain as a sufficient condition for $\kappa_{2} \geq 0$,

$$
\beta_{i}<\left\{\min _{\mathscr{S}_{i}\left(\varepsilon_{i 0}\right)}\left\{\left(q \widehat{-q_{i}}\right) \cdot \widehat{\nabla \beta_{i}}\right\}, \varepsilon_{i 0}\right\} \triangleq \varepsilon_{i 2},
$$


and,

$$
\lambda>2 \sqrt{3} \frac{\max _{\mathscr{F}}\left\{\sum_{j=0, j \neq i}^{M} \frac{1}{E_{j}}\left(\left|\nu_{j}-1\right|+\left\|q-q_{j}\right\|\left\|\nabla \nu_{j}\right\|+\frac{1}{E_{j}}\left\|X_{i j}(q)\right\|\right)\right\}}{\min _{\mathscr{S}_{i}\left(E_{i}\right)}\left\{\nu_{i}\right\}} \triangleq \Lambda_{i 2}^{\prime \prime} .
$$

Finally, letting

completes the proof.

$$
\Lambda_{i 2} \triangleq \max \left\{\lambda_{i 2}^{\prime}, \lambda_{i 2}^{\prime \prime}\right\}
$$

\section{REFERENCES}

1. S. Arimoto and F. Miyazaki, Asymptotic stability of feedback controls for robot manipulators, (Proc. 1st IFAC Sympos. on Robot Control), Barcelona, Spain, 1985.

2. M. Berger, Geometry. I, Springer-Verlag, New York, 1980.

3. J. F. Canny, The complexity of robot motion planning, Ph.D. dissertation, Dept. of Electrical Engineering and Computer Science, M.I.T., May 1987.

4. M. W. Hirsch, Differential topology, Springer-Verlag, New York, 1976.

5. N. Hogan, Impedance control: An approach to manipulation, ASME J. Dynamics Systems, Measurement, and Control 107 (1985), 1-7.

6. W. Hurewicz and H. Wallman, Dimension theory, Princeton Univ. Press, Princeton, N.J., 1962.

7. J. L. Kelley, General topology, Springer-Verlag, New York, 1985.

8. O. Khatib, Real time obstacle avoidance for manipulators and mobile robots, Internat. J. Robotics Res. 5 (1986), 90-99.

9. D. E. Koditschek, A strict global lyapunov function for mechanical systems, Tech. Rep. 8707, Center for Systems Science, Yale Univ., November 1987.

10. _ Robot control systems, (Stuart Shapiro, ed.), Encyclopedia of Artificial Intelligence, Wiley, 1987, pp. 902-923.

11. D. E. Koditschek and E. Rimon, Exact robot navigation using cost functions: The case of distinct spherical boundaries in $E^{n}$, Tech. Rep. 8803, Center for Systems Science, Yale Univ., January 1988.

12. _ Robot navigation functions on manifolds with boundary, Adv. in Appl. Math. (to appear).

13. E. L. Lima, The Jordan-Brouwer separation theorem for smooth hypersurfaces. Amer. Math. Monthly 71 (2) (1988), 39-42.

14. W. S. Massey, Introduction to algebraic topology, Springer-Verlag, New York, 1972.

15. __ Singular homology theory, Springer-Verlag, New York, 1980.

16. J. W. Milnor, Topology from the differentiable viewpoint, The University Press of Virginia, Charlottesville, Va., 1965.

17. M. Morse, The existence of polar non-degenerate functions on differentiable manifolds, Ann. of Math. (2) 71 (1960), 352-383.

18. V. V. Pavlov and A. N. Voronin, The method of potential functions for coding constraints of the external space in an intelligent mobile robot, Soviet Automat. Control 17 (1984).

19. E. Rimon and D. E. Koditschek, The construction of diffeomorphisms for exact robot navigation on star worlds, Tech. Rep. 8809, Center for Systems Science, Yale Univ., July 1988.

20. J. T. Schwartz and M. Sharir, On the "piano movers" problem. II. general techniques for computing topological properties of real algebraic manifolds, Adv. in Appl. Math. 4 (1983), 298-351.

21. I. M. Singer and J. A. Thorpe, Lecture notes on elementary topology and geometry, SpringerVerlag, New York, 1976. 
22. S. Smale, On gradient dynamical systems, Ann. of Math. (2) 74 (1961), 199-206.

23. John A. Thorpe, Elementary topics in differential geometry, Springer-Verlag, New York, 1979.

24. E. Zeidler, Nonlinear functional analysis and its applications. I, Springer-Verlag, New York, 1984.

Department of Electrical Engineering, Center for Systems Science, Yale University, New Haven, Connecticut 06520-1968

Current address, Elon Rimon: Robotics Laboratory, Computer-Science Department, Stanford University, Stanford, California 94305 